\title{
On maximum increase and decrease of Brownian motion
}

\author{
Paavo Salminen \\ Åbo Akademi University \\ Mathematical Department \\ FIN-20500 Åbo, Finland \\ email: phsalmin@abo.fi
}

\author{
Pierre Vallois \\ Université Henri Poincaré \\ Département de Mathématique \\ F-54506 Vandoeuvre les Nancy, France \\ email: vallois@iecn.u-nancy.fr
}

October 22, 2018

\begin{abstract}
The joint distribution of maximum increase and decrease for Brownian motion up to an independent exponential time is computed. This is achieved by decomposing the Brownian path at the hitting times of the infimum and the supremum before the exponential time. It is seen that an important element in our formula is the distribution of the maximum decrease for the three dimensional Bessel process with drift started from 0 and stopped at the first hitting of a given level. From the joint distribution of the maximum increase and decrease it is possible to calculate the correlation coefficient between these at a fixed time and this is seen to be $-0.47936 \ldots$.
\end{abstract}

Dans cet article nous déterminons la loi conjointe de la plus grande montée et de la plus grande descente d'un mouvement brownien arrêté en un temps exponentiel independant. La preuve repose sur la décomposition de la trajectoire brownienne aux instants où le processus atteint son maximum, resp. son minimum, avant le temps exponentiel. La loi de la plus grande descente d'un processus de Bessel, de dimension trois, issu de 0 et arrêté lorsqu'il atteint un niveau fixé, joue également 
un rôle important. Le coefficient de corrélation linéaire de la grande montée et de la plus grande descente d'un mouvement brownien arrêté en temps fixe est déterminé : $-0.47936 \ldots$.

Keywords: $h$-transform, time reversal, path decompositions, Brownian motion with drift, excursion process, maximum process, Itô measure, maximum drawdown, covariance, Catalan's constant.

AMS Classification: 60J60, 60J65, 60G17, 62P05. 


\section{Introduction and notation}

1. In this paper we are interested in the joint distribution of the maximum increase and decrease for a standard Brownian motion, for short BM. Let us start with some notation. Let $\Omega:=\mathcal{C}\left(\mathbf{R}_{+}, \mathbf{R}\right)$ be the space of continuous functions $\omega: \mathbf{R}_{+} \mapsto \mathbf{R}$ and $X_{t}(\omega)=\omega(t), t \geq 0$, the coordinate mappings. With every $\omega$ we associate its lifetime $\zeta(\omega) \in(0, \infty]$ and consider $X_{t}$ to be defined for $t<\zeta(\omega)$. The standard notation $\mathcal{F}_{t}$ is used for the $\sigma$-algebra generated by the coordinate mappings up to time $t$, and we set $\mathcal{F}:=\mathcal{F}_{\infty}$.

Further, $\mathbf{P}_{x}^{\mu}$ and $\mathbf{E}_{x}^{\mu}$ denote the probability measure and the expectation operator on $(\Omega, \mathcal{F})$ under which the coordinate process $X=\left\{X_{t}: t \geq 0\right\}$ is a Brownian motion with drift $\mu$ started from $x$, for short $\operatorname{BM}(\mu)$. For simplicity, $\mathbf{P}_{x}$ and $\mathbf{E}_{x}$ stand for the corresponding objects for BM.

The maximum increase before time $t$ is defined as

$$
D_{t}^{+}:=\sup _{0 \leq u \leq v \leq t}\left(X_{v}-X_{u}\right)
$$

and, analogously, the maximum decrease

$$
D_{t}^{-}:=\sup _{0 \leq u \leq v \leq t}\left(X_{u}-X_{v}\right)
$$

Notice that, e.g.,

$$
D_{t}^{-}=\sup _{0 \leq v \leq t}\left(\sup _{0 \leq u \leq v} X_{u}-X_{v}\right) .
$$

Using the Lévy isomorphism, i.e., under $\mathbf{P}_{0}$

$$
\left\{X_{v}^{+}:=\sup _{0 \leq u \leq v} X_{u}-X_{v}: v \geq 0\right\} \stackrel{(d)}{=}\left\{\left|X_{v}\right|: v \geq 0\right\}
$$

where $\stackrel{(d)}{=}$ means "to be identical in law with", it follows from identity (1.3) that

$$
\mathbf{P}_{0}\left(D_{t}^{-}>a\right)=\mathbf{P}_{0}\left(H_{a} \wedge H_{-a}<t\right)
$$

with

$$
H_{b}:=\inf \left\{t: X_{t}=b\right\}, \quad b \in \mathbf{R},
$$

the first hitting time of $b$ (in the canonical setting) with the usual convention that $H_{b}=+\infty$ if the set in the braces is empty. From the equality (1.5) 
applying, e.g., 4] 1.3.0.2 p. 212, and 3.1.1.4 p. 333 we obtain

$$
\begin{aligned}
\mathbf{P}_{0}\left(D_{t}^{-}>a\right) & =\sum_{k=-\infty}^{+\infty}(-1)^{k} \int_{0}^{t} d s \frac{(2 k+1) a}{\sqrt{2 \pi} s^{3 / 2}} \mathrm{e}^{-(2 k+1)^{2} a^{2} / 2 s} \\
& =1-\frac{1}{\sqrt{2 \pi t}} \sum_{k=-\infty}^{+\infty}(-1)^{k} \int_{-a}^{a} d x\left(\mathrm{e}^{-(x+4 k a)^{2} / 2 t}-\mathrm{e}^{-(x+4 k a+2 a)^{2} / 2 t}\right) .
\end{aligned}
$$

Notice also that due to the symmetry of standard Brownian motion we have

$$
\left\{D_{t}^{-}: t \geq 0\right\} \stackrel{(\mathrm{d})}{=}\left\{D_{t}^{+}: t \geq 0\right\}
$$

and this holds under $\mathbf{P}_{x}$ for any $x \in \mathbf{R}$. We refer to Douady, et al. [6] for results concerning the distribution of maximum increase and related functionals up to a fixed time in the case of Brownian motion.

For Brownian motion with drift the result corresponding to (1.4) states that under $\mathbf{P}_{x}^{\mu}$ the process $\left\{X_{v}^{+}: v \geq 0\right\}$ is a reflected Brownian motion on $\mathbf{R}_{+}$with drift $-\mu$, for short $\operatorname{RBM}(-\mu)$, (see, e.g., Harrison [7] p. 49, and McKean [9] p. 71), more precisely, it is a diffusion on $\mathbf{R}_{+}$with basic characteristics as given in 4 A1.16 p. 129. The probability measure on $(\Omega, \mathcal{F})$ associated with $X^{+}$(under $\left.\mathbf{P}^{\mu}\right)$ is denoted by $\mathbf{P}^{-\mu,+}$. Clearly, we have for a given $a>0$

$$
\mathbf{P}_{0}^{\mu}\left(D_{t}^{-}>a\right)=\mathbf{P}_{0}^{-\mu,+}\left(H_{a}<t\right)
$$

Similarly, defining

$$
X_{v}^{-}:=X_{v}-\inf _{0 \leq u \leq v} X_{u}
$$

it holds under $\mathbf{P}^{\mu}$ that the process $X^{-}$is a $\operatorname{RBM}(\mu)$. Letting $\mathbf{P}^{\mu,+}$ denote the measure associated with $X^{-}$we have

$$
\mathbf{P}_{0}^{\mu}\left(D_{t}^{+}>a\right)=\mathbf{P}_{0}^{\mu,+}\left(H_{a}<t\right) .
$$

For an explicit expression of the $\mathbf{P}^{\mu}$-distribution of $D_{t}^{-}$, see Dominé [5] where the method based on spectral representations is used. In Magdon-Ismail et.al. 8) formulas for the mean of $D_{t}^{-}$are derived.

2. Unfortunately we are not able to determine explicitly the distribution of $\left(D_{t}^{+}, D_{t}^{-}\right)$, but replacing $t$ by $T$, that is, an exponentially distributed random variable independent of $X$ with mean $1 / \lambda$, allows us to find the $\mathbf{P}$ distribution of $\left(D_{T}^{+}, D_{T}^{-}\right)$, see Proposition 4.5 and 4.6. We remark that the 
marginal $\mathbf{P}^{\mu}$-distributions of $D_{T}^{+}$and $D_{T}^{-}$are easily computed from (1.7) and (1.6), respectively. Indeed, using standard diffusion theory and some explicit formulas (see e.g. [4] p. 18 and 129) yield

$$
\mathbf{P}_{0}^{\mu}\left(D_{T}^{-}>a\right)=\mathbf{E}_{0}^{-\mu,+}\left(\exp \left(-\lambda H_{a}\right)\right)=1 / \psi_{\lambda}(a ;-\mu)
$$

and

$$
\mathbf{P}_{0}^{\mu}\left(D_{T}^{+}>a\right)=1 / \psi_{\lambda}(a ; \mu)
$$

with

$$
\psi_{\lambda}(a ; \nu):=\mathrm{e}^{-\nu a}\left(\operatorname{ch}\left(a \sqrt{2 \lambda+\nu^{2}}\right)+\frac{\nu}{\sqrt{2 \lambda+\nu^{2}}} \operatorname{sh}\left(a \sqrt{2 \lambda+\nu^{2}}\right)\right) .
$$

In our approach for finding the joint distribution we consider first the case where the infimum is attained before the supremum. In this case it is clear that the maximum increase is nothing but the difference of the supremum and the infimum, and, in a sense, we have reduced the problem to the problem for finding the distribution of the maximum decrease. The opposite case where the supremum is attained before the infimum is clearly treated using symmetry.

It is natural when the infimum is attained before the supremum to decompose the exponentially stopped Brownian path into three parts:

o the first part is up to the hitting time of the infimum,

o the second part is from the hitting time of the infimum to the hitting time of the supremum

o the third part is from the hitting time of the supremum to the exponential time.

We prove in Theorem 3.5 that these three parts are conditionally independent given the infimum and the supremum, and find their distributions in terms of the three dimensional Bessel processes with drift. Our approah is mainly based on the $h$-transform techniques, excursion theory and path decompositions of Brownian motion with drift.

The above described path decomposition up to $T$ permits us to determine the joint distribution of $\left(D_{T}^{+}, D_{T}^{-}\right)$since now $D_{T}^{+}=S_{T}-I_{T}$ and, under this decomposition, $D_{T}^{-}$is the maximum of the maximum decreases of the three conditionally independent fragments. To find the distribution of the 
maximum decrease for the first and the third part is fairly straightforward diffusion theory. To compute the maximum decrease for the second part is equivalent for finding the distribution of the maximum decrease for a three dimensional Bessel process with drift (see Proposition 2.5).

Although the distribution and the density function of $\left(D_{T}^{+}, D_{T}^{-}\right)$are complicated it is possible to determine by the scaling property of BM the covariance between $D_{t}^{+}$and $D_{t}^{-}$and this is given by

$$
\mathbf{E}\left(D_{t}^{+} D_{t}^{-}\right)=(1-2 \log 2+2 \beta(2)) t
$$

where

$$
\beta(2):=\sum_{k=0}^{\infty}(-1)^{k}(2 k+1)^{-2}=0.91596 \ldots
$$

is Catalan's constant. Hence, the correlation coefficient $\rho$ between $D_{T}^{+}$and $D_{T}^{-}$is easily obtained to be

$$
\rho:=\frac{\mathbf{E}\left(D_{t}^{+} D_{t}^{-}\right)-\left(\mathbf{E}\left(D_{t}^{+}\right)\right)^{2}}{\operatorname{Var}\left(D_{t}^{+}\right)}=-0.47936 \ldots
$$

3. One motivation to study the maximum decrease and increase comes from mathematical finance where the maximum decrease, also called maximum drawdown (MDD), is used to quantify the riskyness of a stock or any other asset. Related measures used hereby are e.g. the recovery time from MDD and the duration of MDD. Our interest to the problem discussed in the paper was arised by Gabor Szekely who asked for an expression for the covariance between $D_{t}^{+}$and $D_{t}^{-}$.

4. The paper is organised so that in the next section we find the distribution of the maximum decrease of a stopped Brownian motion with positive drift. In fact, we compute this distribution under the restriction that the process does not hit some negative level, and proceed from here to the distribution of the maximum decrease for a three dimensional Bessel process with drift. In the third section path decompositions are discussed. To prove our main path decomposition Theorem 3.5, we first prove a decomposition of the Brownian trajectory $\left\{B_{t}: t \leq T\right\}$ conditionally on $I_{T}$ (see Theorem 3.2). The fourth section is devoted to computation and analysis of the law of $\left(D_{T}^{-}, D_{T}^{+}\right)$. 


\section{Maximum decrease for stopped Brownian mo- tion with drift}

According to (1.2), the maximum decrease up to the first hitting time of a given level $\beta$ is

$$
D_{H_{\beta}}^{-}:=\sup \left\{X_{u}-X_{v}: 0 \leq u \leq v \leq H_{\beta}\right\}
$$

In this section we consider the $\mathbf{P}^{\mu}$-distribution of $D_{H_{\beta}}^{-}$under some additional conditions and conditioning. Recall that

$$
S^{\mu}(x):=\frac{1}{2 \mu}\left(1-\mathrm{e}^{-2 \mu x}\right)
$$

is the scale function of $\operatorname{BM}(\mu)$ and for $a<x<b$

$$
\mathbf{P}_{x}^{\mu}\left(H_{a}<H_{b}\right)=\frac{S^{\mu}(b)-S^{\mu}(x)}{S^{\mu}(b)-S^{\mu}(a)} .
$$

Proposition 2.1. For all nonnegative $\alpha, \beta$, and $u$

$$
\mathbf{P}_{0}^{\mu}\left(D_{H_{\beta}}^{-}<u, H_{\beta}<H_{-\alpha}\right)=\left\{\begin{array}{l}
\exp \left(-\frac{\beta}{S^{-\mu}(u)}\right), u \leq \alpha, \\
\frac{S^{\mu}(\alpha)}{S^{\mu}(u)} \exp \left(-\frac{\beta+\alpha-u}{S^{-\mu}(u)}\right), \\
\frac{S^{\mu}(\alpha)}{S^{\mu}(\alpha+\beta)}, \quad \alpha \leq \alpha+\beta,
\end{array}\right.
$$

In particular,

$$
\mathbf{P}_{0}^{\mu}\left(D_{H_{\beta}}^{-}<u\right)=\exp \left(-\frac{\beta}{S^{\mu}(u)}\right) .
$$

For standard Brownian motion, i.e., $\mu=0$, the above formulas hold with $S^{0}(u)=u$.

Proof. We assume that $X_{0}=0$, and define for $a>0$

$$
H_{a+}:=\inf \left\{t: X_{t}>a\right\} .
$$


For a given $a>0$, in the case $H_{a+}>H_{a}$, let

$$
\Xi_{a}^{+}(u):=a-X_{u+H_{a}}, \quad 0 \leq u<H_{a+}-H_{a},
$$

and, if $H_{a+}=H_{a}$, take $\Xi_{a}^{+}:=\partial$, where $\partial$ is some fictious (cemetary) state. The process

$$
\Xi^{+}=\left\{\Xi_{a}^{+}: a \geq 0\right\}
$$

is called the excursion process, associated with $X$, for excursions under the running maximum. Let, further,

$$
M_{a}:=\sup \left\{\Xi_{a}^{+}(u): 0 \leq u<H_{a+}-H_{a}\right\} .
$$

An obvious but important fact is that

$$
D_{H_{\beta}}^{-}=\sup _{a<\beta} M_{a} .
$$

Introduce also for $u>0$

$$
\xi_{u}:=\inf \left\{a \geq 0: M_{a}>u\right\},
$$

and

$$
\xi_{u}^{\circ}:=\inf \left\{a \geq 0: a-M_{a}<-u\right\} .
$$

Then it holds for positive $\alpha, \beta$, and $u$

$$
\left\{D_{H_{\beta}}^{-} \leq u\right\}=\left\{\forall a \in(0, \beta): M_{a} \leq u\right\}=\left\{\xi_{u} \geq \beta\right\},
$$

and

$$
\left\{H_{\beta}<H_{-\alpha}\right\}=\left\{\forall a \in(0, \beta): a-M_{a}>-\alpha\right\}=\left\{\xi_{\alpha}^{\circ}>\beta\right\} ;
$$

hence, for $0<u<\alpha+\beta$

$$
\mathbf{P}_{0}^{\mu}\left(D_{H_{\beta}}^{-}<u, H_{\beta}<H_{-\alpha}\right)=\mathbf{P}_{0}^{\mu}\left(\xi_{u}>\beta, \xi_{\alpha}^{\circ}>\beta\right) .
$$

Since $X^{+}$under $\mathbf{P}^{\mu}$ is identical in law with $\operatorname{RBM}(-\mu)$ it follows that the excursion process $\Xi^{+}$is identical in law with the usual excursion process of $\operatorname{RBM}(-\mu)$ for excursions from 0 to 0 . Consequently, see Pitman and Yor [11,

$$
\Pi=\left\{\left(a, M_{a}\right): a \geq 0\right\}
$$


is a homogeneous Poisson point process with the characteristic measure

$$
\nu(d a, d m)=d a n(d m),
$$

where for $m>0$

$$
n((m,+\infty))=1 / S^{-\mu}(m)
$$

Introduce the sets

$$
A:=[0, \beta) \times[u,+\infty) \text { and } B:=\{(a, m): 0 \leq a<\beta, a-m<-\alpha\},
$$

and let $N$ denote the counting measure associated with $\Pi$. Now we have

$$
\begin{aligned}
\mathbf{P}_{0}^{\mu}\left(\xi_{u}>\beta, \xi_{\alpha}^{\circ}>\beta\right) & =\mathbf{P}_{0}^{\mu}(N(A \cup B)=0) \\
& =\exp (-\nu(A \cup B)) .
\end{aligned}
$$

It is straightforward to compute $\nu(A \cup B)$ for different values on $\alpha, \beta$, and $u$, and we leave this to the reader. Consequently, by (2.4), the claimed formula is obtained.

Obviously, $D_{H_{\beta}}^{+} \geq \beta$ and for $z>0$

$$
\left\{D_{H_{\beta}}^{+}-\beta<z\right\}=\left\{H_{\beta}<H_{-z}\right\} .
$$

Consequently, we have from Proposition 2.1 the following corollary giving an expression for the joint distribution of $D_{H_{\beta}}^{+}$and $D_{H_{\beta}}^{-}$. Notice that

$$
D_{H_{\beta}}^{-} \leq D_{H_{\beta}}^{+} \leq D_{H_{\beta}}^{-}+\beta
$$

explaining the three cases below.

Corollary 2.2. For $v \geq \beta$ and $\mu \geq 0$

$$
\mathbf{P}_{0}^{\mu}\left(D_{H_{\beta}}^{-}<u, D_{H_{\beta}}^{+}<v\right)= \begin{cases}\exp \left(-\frac{\beta}{S^{-\mu}(u)}\right), \quad u \leq v-\beta, \\ \frac{S^{\mu}(v-\beta)}{S^{\mu}(u)} \exp \left(-\frac{v-u}{S^{-\mu}(u)}\right), \\ v-\beta \leq u \leq v, \\ \frac{S^{\mu}(v-\beta)}{S^{\mu}(v)}, & v \leq u,\end{cases}
$$

For standard Brownian motion, i.e., $\mu=0$, the above formulas hold with $S^{0}(u)=u$. 
We proceed by developing the result in Proposition 2.1 for a 3-dimensional Bessel process with drift $\mu>0$, for short $\operatorname{BES}(3, \mu)$. We recall that $\operatorname{BES}(3, \mu)$ is a linear diffusion with the generator

$$
\mathcal{G}^{R, \mu}=\frac{1}{2} \frac{d^{2}}{d x^{2}}+\mu \operatorname{coth}(\mu x) \frac{d}{d x}, \quad x>0 .
$$

The notation $\mathbf{Q}_{x}^{\mu}$ is used for the probability measure on the canonical space $\Omega$ associated with $\operatorname{BES}(3, \mu)$ when started from $x \geq 0$. In the case $\mu=0$ the corresponding measure is simply denoted by $\mathbf{Q}_{x}$ and the generator is given by

$$
\mathcal{G}^{R}=\frac{1}{2} \frac{d^{2}}{d x^{2}}+\frac{1}{x} \frac{d}{d x}, \quad x>0 .
$$

The following lemma is a fairly well known example on $h$-transforms. To make the presentation more self contained we give a short proof. It is also interesting to compare the result with Lemma 3.1 in the next section.

Lemma 2.3. Let $0<x<y$ be given. The Brownian motion with drift $\mu$ started from $x>0$, killed at the first hitting time of $y$, and conditioned to hit $y$ before 0 is identical in law with a 3-dimensional Bessel process with drift $|\mu|$ started from $x$ and killed at the first hitting time of $y$.

Proof. In our canonical space of continuous functions with $X_{0}=x$ we have for a given $t>0$

$$
\left\{t<H_{y}<H_{0}\right\}=\left\{t<H_{y} \wedge H_{0}, H_{y} \circ \theta_{t}<H_{0} \circ \theta_{t}\right\},
$$

where $\theta$. is the usual shift operator, i.e., $X_{s} \circ \theta_{t}=X_{s+t}$. Hence, for any $A_{t} \in \mathcal{F}_{t}$,

$$
\begin{aligned}
\mathbf{P}_{x}^{\mu}\left(A_{t}, t<\right. & \left.H_{y} \mid H_{y}<H_{0}\right) \\
& =\mathbf{P}_{x}^{\mu}\left(A_{t}, t<H_{y} \wedge H_{0}, H_{y} \circ \theta_{t}<H_{0} \circ \theta_{t}\right) / \mathbf{P}_{x}^{\mu}\left(H_{y}<H_{0}\right) \\
& =\mathbf{E}_{x}^{\mu}\left(h_{1}\left(X_{t}\right) ; A_{t}, t<H_{y} \wedge H_{0}\right) / h_{1}(x)
\end{aligned}
$$

by the Markov property, where

$$
h_{1}(x):=\mathbf{P}_{x}^{\mu}\left(H_{y}<H_{0}\right)=\frac{S^{\mu}(x)-S^{\mu}(0)}{S^{\mu}(y)-S^{\mu}(0)}=\frac{1-\mathrm{e}^{-2 \mu x}}{1-\mathrm{e}^{-2 \mu y}} .
$$


Consequently, the desired conditioning can be realized by taking the Doob $h$-transform (with $h=h_{1}$ ) of the Brownian motion with drift $\mu$ killed at $H_{y} \wedge H_{0}$. The generator of the $h$-transform is

$$
\mathcal{G}^{h_{1}}:=\frac{1}{2} \frac{d^{2}}{d x^{2}}+\mu \frac{d}{d x}+\frac{h^{\prime}(x)}{h(x)} \frac{d}{d x}
$$

which is easily seen to coincide with (2.5) with $|\mu|$ instead of $\mu$.

Remark 2.4. Analogously as above, it can be proved that $B M(\mu)$ started from $x>0$ and conditioned not to hit 0 is identical in law with $\operatorname{BES}(3,|\mu|)$ started from $x$.

Proposition 2.5. For $\beta>u>0$

$$
\mathbf{Q}_{0}^{\mu}\left(D_{H_{\beta}}^{-}<u\right)=\frac{S^{-\mu}(\beta)}{S^{-\mu}(u)} \exp \left(-\frac{\beta-u}{S^{-\mu}(u)}-2 \mu(\beta-u)\right) .
$$

For the 3-dimensional Bessel process without drift, i.e., $\mu=0$, the above formula holds with $S^{0}(u)=u$.

Proof. Note that under $\mathbf{Q}_{0}^{\mu}$, it holds a.s. on $\left\{D_{H_{\beta}}^{-}>u\right\}$ that

$$
D_{H_{\beta}}^{-}=D_{H_{\beta}}^{-} \circ \theta_{H_{u}} .
$$

Therefore, applying the strong Markov property at time $H_{u}$ yields

$$
\mathbf{Q}_{0}^{\mu}\left(D_{H_{\beta}}^{-}>u\right)=\mathbf{Q}_{u}^{\mu}\left(D_{H_{\beta}}^{-}>u\right)
$$

and from Lemma 2.3

$$
\begin{aligned}
\mathbf{Q}_{u}^{\mu}\left(D_{H_{\beta}}^{-}>u\right) & =\mathbf{P}_{u}^{\mu}\left(D_{H_{\beta}}^{-}>u \mid H_{\beta}<H_{0}\right) \\
& =\mathbf{P}_{u}^{\mu}\left(D_{H_{\beta}}^{-}>u, H_{\beta}<H_{0}\right) / \mathbf{P}_{u}^{\mu}\left(H_{\beta}<H_{0}\right) \\
& =\mathbf{P}_{0}^{\mu}\left(D_{H_{\beta-u}}^{-}>u, H_{\beta-u}<H_{-u}\right) / \mathbf{P}_{0}^{\mu}\left(H_{\beta-u}<H_{-u}\right) .
\end{aligned}
$$

The proof is now easily completed from Proposition 2.1. 


\section{Path decompositions}

The main path decomposition results presented in this section are stated in Theorems 3.2 and 3.5. In the first one we consider the decomposition at the global infimum and the second one gives, roughly speaking, the decomposition of the post part of the previous decomposition at its global supremum.

We begin with by stating the following lemma which is proved similarly as Lemma 2.3

Lemma 3.1. Let $0<x<y$ be given. The Brownian motion started from $x>0$, killed at the first hitting time of $y$, and conditioned by the event $H_{y}<H_{0} \wedge T$, where $T$ is an exponentially distributed random variable with parameter $\lambda$ independent of the Brownian motion, is identical in law with $\operatorname{BES}(3, \sqrt{2 \lambda})$ started from $x$ and killed at the first hitting time of $y$.

Proof. We adapt the proof of Lemma 2.3 to our new situation. For $t>0$ we have

$$
\left\{t<H_{y}<H_{0} \wedge T\right\}=\left\{t<H_{y} \wedge H_{0} \wedge T, H_{y} \circ \theta_{t}<\left(H_{0} \wedge T\right) \circ \theta_{t}\right\}
$$

Hence, using the memoryless property of $T$ we get for $A_{t} \in \mathcal{F}_{t}$

$$
\begin{aligned}
\mathbf{P}_{x}\left(A_{t}, t<\right. & \left.H_{y} \mid H_{y}<H_{0} \wedge T\right) \\
& =\mathbf{E}_{x}\left(h_{2}\left(X_{t}\right) ; A_{t}, t<H_{y} \wedge H_{0} \wedge T\right) / h_{2}(x)
\end{aligned}
$$

with

$$
\begin{aligned}
h_{2}(x) & =\mathbf{P}_{x}\left(H_{y}<H_{0} \wedge T\right)=\mathbf{P}_{x}\left(H_{y}<H_{0}, H_{y}<T\right) \\
& =\operatorname{sh}(x \sqrt{2 \lambda}) / \operatorname{sh}(y \sqrt{2 \lambda})
\end{aligned}
$$

(see [4] 1.3.0.5(b) p.212).

Consequently, the desired conditioning can be realized by taking the Doob $h$-transform (with $h=h_{2}$ ) of a Brownian motion killed at time $H_{y} \wedge H_{0} \wedge T$. The generator of the $h$-transform can be computed in the usual way, and is seen to coincide with the generator of $\operatorname{BES}(3, \sqrt{2 \lambda})$ (see (2.5) ).

We let, throughout the paper, $T$ denote an exponentially with parameter $\lambda$ distributed random variable independent of $X$ under $\mathbf{P}_{0}$, and define

$$
I_{T}:=\inf \left\{X_{t}: 0 \leq t \leq T\right\} \quad, \quad S_{T}:=\sup \left\{X_{t}: 0 \leq t \leq T\right\}
$$


and

$$
H_{I}:=\inf \left\{t: X_{t}=I_{T}\right\} \quad, \quad H_{S}:=\inf \left\{t: X_{t}=S_{T}\right\} .
$$

Next we discuss the path decomposition at the global infimum for Brownian motion killed at $T$. If nothing else is stated the coordinate process is considered under $\mathbf{P}_{0}$.

Theorem 3.2. 1. The processes $\left\{X_{t}: 0 \leq t<H_{I}\right\}$ and $\left\{X_{T-t}-X_{T}: 0 \leq\right.$ $\left.t<T-H_{I}\right\}$ are independent and identically distributed.

2. Given $I_{T}=a$

1. the pre- $H_{I}$ process $\left\{X_{t}: 0 \leq t<H_{I}\right\}$ and the post- $H_{I}$ process $\left\{X_{H_{I}+t}\right.$ : $\left.0 \leq t<T-H_{I}\right\}$ are independent.

2. the pre- $H_{I}$ process is identical in law with a $B M(-\sqrt{2 \lambda})$ killed when it hits a,

3. the post- $H_{I}$ process is identical in law with the diffusion $Z$ started from $a$ and having the generator

$$
\mathcal{G}^{Z} u(x)=\frac{1}{2} u^{\prime \prime}(x)+\frac{h_{3}^{\prime}(x-a)}{h_{3}(x-a)} u^{\prime}(x)-\frac{\lambda}{h_{3}(x-a)} u(x),
$$

where $x>a$ and

$$
h_{3}(y):=\mathbf{P}_{y}\left(T<H_{0}\right)=1-\mathrm{e}^{-y \sqrt{2 \lambda}}, \quad y>0 .
$$

Moreover, $Z$ is the Doob h-transform with $h=h_{3}(\cdot-a)$ of BM killed at time $T \wedge H_{a}$.

Proof. a) We have two different proofs: the first one is "direct" in a sense that we compute the conditional finite dimensional distributions, the second one relies on excursion theory of Brownian motion. From our point of view the both proofs contain interesting elements which motivates the presentation of both of these.

b) We begin with the direct proof of claim 2. Define for $s<t$

$$
I_{s, t}:=\inf \left\{X_{u}: s \leq u \leq t\right\}, \quad I_{t}:=I_{0, t}
$$

and

$$
H_{I_{s, t}}:=\inf \left\{u \in(s, t): X_{u}=I_{s, t}\right\}, \quad H_{I_{t}}:=H_{I_{0, t}} .
$$


Let $u, v$, and $t$ be given such that $0<u<v<t$. For positive integers $n$ and $m$ introduce $0<u_{1}<\cdots<u_{n}<u$ and $0<v_{1}<\cdots<v_{m}$ with $v_{m}+v<t$. Define also

$$
A_{n}:=\left\{X_{u_{1}} \in d x_{1}, \ldots, X_{u_{n}} \in d x_{n}\right\}
$$

and

$$
B_{m}:=\left\{X_{v_{1}} \in d y_{1}, \ldots, X_{v_{m}} \in d y_{m}\right\} .
$$

Consider now for $u<s<v$

$$
\begin{aligned}
& \mathbf{P}_{0}\left(A_{n}, I_{t} \in d a, H_{I_{t}} \in d s, B_{m} \circ \theta_{v}, X_{t} \in d z\right) \\
& =\mathbf{P}_{0}\left(A_{n}, I_{u}>a, I_{u, v} \in d a, H_{I_{u, v}} \in d s, I_{v, t}>a, B_{m} \circ \theta_{v}, X_{t} \in d z\right) \\
& =\mathbf{P}_{0}\left(A_{n}, I_{u}>a ; \mathbf{P}_{0}\left(I_{u, v} \in d a, H_{I_{u, v}} \in d s, I_{v, t}>a, B_{m} \circ \theta_{v}, X_{t} \in d z \mid \mathcal{F}_{u}\right)\right) .
\end{aligned}
$$

Further,

$$
\begin{aligned}
& \mathbf{P}_{0}\left(I_{u, v} \in d a, H_{I_{u, v}} \in d s, I_{v, t}>a, B_{m} \circ \theta_{v}, X_{t} \in d z \mid \mathcal{F}_{u}\right) \\
& \quad=\mathbf{P}_{0}\left(I_{u, v} \in d a, H_{I_{u, v}} \in d s ; \mathbf{P}_{0}\left(I_{v, t}>a, B_{m} \circ \theta_{v}, X_{t} \in d z \mid \mathcal{F}_{v}\right) \mid \mathcal{F}_{u}\right) \\
& \quad=\mathbf{P}_{0}\left(I_{u, v} \in d a, H_{I_{u, v}} \in d s ; \mathbf{P}_{0}\left(I_{v, t}>a, B_{m} \circ \theta_{v}, X_{t} \in d z \mid X_{v}\right) \mid X_{u}\right)
\end{aligned}
$$

by the Markov property. Letting $p^{+}$denote the transition density (with respect to $2 d x$ ) of BM killed when it hits $a$ and writing $X_{v}=z_{2}$ we have

$$
\begin{aligned}
\mathbf{P}_{0}\left(I_{v, t}>a, B_{m}\right. & \left.\circ \theta_{v}, X_{t} \in d z \mid X_{v}\right) \\
& =p^{+}\left(v_{1} ; z_{2}, y_{1}\right) 2 d y_{1} \cdots p^{+}\left(t-v-v_{m} ; y_{m}, z\right) 2 d z \\
& =: F_{v_{1}, \ldots, v_{m}, t-v}\left(z_{2}, y_{1}, \ldots, y_{m}, z\right) 2 d y_{1} \ldots 2 d z .
\end{aligned}
$$

Introduce

$$
\eta_{x}(a, \alpha):=\mathbf{P}_{x}\left(H_{a} \in d \alpha\right) / d \alpha,
$$

and recall the formula due to Lévy

$$
\begin{aligned}
\mathbf{P}_{x}\left(I_{\beta} \in d a, H_{I_{\beta}}\right. & \left.\in d \alpha, X_{\beta} \in d z\right) \\
& =\eta_{x}(a, \alpha) \eta_{z}(a, \beta-\alpha) d \alpha 2 d z d a, \quad \alpha<\beta .
\end{aligned}
$$

Applying (3.6) and putting $X_{u}=z_{1}$ we obtain

$$
\begin{aligned}
& \mathbf{P}_{0}\left(I_{u, v} \in d a, H_{I_{u, v}} \in d s ; \mathbf{P}_{0}\left(I_{v, t}>a, B_{m} \circ \theta_{v}, X_{t} \in d z \mid \mathcal{F}_{v}\right) \mid \mathcal{F}_{u}\right) \\
&=\int_{a}^{\infty} 2 d z_{2} \eta_{z_{1}}(a, s-u) \eta_{z_{2}}(a, v-s) d a d s \\
& \quad \quad \times F_{v_{1}, \ldots, v_{m}, t-v}\left(z_{2}, y_{1}, \ldots, y_{m}, z\right) 2 d y_{1} \ldots 2 d y_{m} 2 d z
\end{aligned}
$$


and, finally,

$$
\begin{aligned}
& \mathbf{P}_{0}\left(A_{n}, I_{t} \in d a, H_{I_{t}} \in d s, B_{m} \circ \theta_{v}, X_{t} \in d z\right) \\
& =\int_{a}^{\infty} 2 d z_{1} \int_{a}^{\infty} 2 d z_{2} p^{+}\left(u_{1} ; 0, x_{1}\right) 2 d x_{1} \cdots p^{+}\left(u-u_{n} ; x_{n}, z_{1}\right) \\
& \quad \times \eta_{z_{1}}(a, s-u) \eta_{z_{2}}(a, v-s) d a d s \\
& \quad \times F_{v_{1}, \ldots, v_{m}, t-v}\left(z_{2}, y_{1}, \ldots, y_{m}, z\right) 2 d y_{1} \ldots 2 d y_{m} 2 d z \\
& =\int_{a}^{\infty} 2 d z_{1} \int_{a}^{\infty} 2 d z_{2} F_{u_{1}, \ldots, u_{n}, u}\left(0, x_{1}, \ldots, x_{n}, z_{1}\right) 2 d x_{1} \ldots 2 d x_{n} \\
& \quad \times \eta_{z_{1}}(a, s-u) \eta_{z_{2}}(a, v-s) d a d s \\
& \quad \times F_{v_{1}, \ldots, v_{m}, t-v}\left(z_{2}, y_{1}, \ldots, y_{m}, z\right) 2 d y_{1} \ldots 2 d y_{m} 2 d z
\end{aligned}
$$

Replacing in (3.6) the deterministic time $\beta$ with the exponential time $T$ yields for $a<0$ and $a<z$

$$
\begin{aligned}
\mathbf{P}_{0}\left(I_{T} \in d a, H_{I}\right. & \left.\in d s, X_{T} \in d z\right) \\
& =\eta_{0}(a, s) \lambda \mathrm{e}^{-\lambda s} \mathbf{E}_{z}\left(\mathrm{e}^{-\lambda H_{a}}\right) d s 2 d z d a,
\end{aligned}
$$

and, further,

$$
\mathbf{P}_{0}\left(I_{T} \in d a, H_{I} \in d s\right)=\sqrt{2 \lambda} \mathrm{e}^{-\lambda s} \eta_{0}(a, s) d a d s .
$$

We operate similarly in (3.7), i.e., introduce the exponential time $T$ in place of $t$. After this we integrate over $z$, and divide with the expression on the r.h.s. in (3.9) and obtain for $u<s<v$

$$
\begin{aligned}
\mathbf{P}_{0}\left(A_{n}, B_{m} \circ \theta_{v} \mid\right. & \left.I_{T}=a, H_{I}=s\right) \\
= & \widehat{F}_{u_{1}, \ldots, u_{n}}\left(x_{1}, \ldots, x_{n} ; a, s\right) 2 d x_{1} \ldots 2 d x_{n} \\
& \times \widehat{G}_{v_{1}, \ldots, v_{m}}\left(y_{1}, \ldots, y_{m} ; a ; s, v\right) 2 d y_{1} \ldots 2 d y_{m}
\end{aligned}
$$

with

$$
\begin{aligned}
& \widehat{F}_{u_{1}, \ldots, u_{n}}\left(x_{1}, \ldots, x_{n} ; a ; s\right) \\
& \quad=\widehat{p}^{+}\left(u_{1} ; 0, x_{1}\right) \cdots \cdots \widehat{p}^{+}\left(u_{n}-u_{n-1} ; x_{n-1}, x_{n}\right) \frac{\widehat{\eta}_{x_{n}}\left(a, s-u_{n}\right)}{\widehat{\eta}_{0}(a, s)}
\end{aligned}
$$

and

$$
\begin{aligned}
\widehat{G}_{v_{1}, \ldots, v_{n}}\left(y_{1}, \ldots, y_{n} ; a ; s, v\right) & =\frac{\widehat{\eta}_{y_{1}}\left(a, v-s+v_{1}\right)}{\sqrt{2 \lambda}} \\
& \times \widehat{p}^{+}\left(v_{2}-v_{1} ; y_{1}, y_{2}\right) \cdots \widehat{p}^{+}\left(v_{m}-v_{m-1} ; y_{m-1}, y_{m}\right) h_{3}\left(y_{m}-a\right)
\end{aligned}
$$


where $h_{3}$ is as in (3.2) and

$$
\widehat{p}^{+}(\alpha ; x, y):=\mathrm{e}^{-\lambda \alpha} p^{+}(\alpha ; x, y), \quad \widehat{\eta}_{x}(a, \alpha):=\mathrm{e}^{-\lambda \alpha} \eta_{x}(a, \alpha) .
$$

Because

$$
\widehat{\eta}_{x}(a, \alpha)=\mathbf{P}_{x}\left(H_{a} \in d \alpha, H_{a}<T\right) / d \alpha
$$

it is seen from (3.11) that $F$ describes the finite dimensional distributions of $X$ conditioned to hit $a$ at time $s$ before $T$. For the claim concerning the post-process we remark first that (3.12) gives finite dimensional distributions of the announced $h$-transform started from $a$ since

$$
\frac{\widehat{\eta}_{y_{1}}\left(a, v-s+v_{1}\right)}{\sqrt{2 \lambda}}=\lim _{x \downarrow a} \frac{\widehat{p}^{+}\left(v-s+v_{1} ; x, y_{1}\right)}{h_{3}(x-a)} .
$$

Next notice that proceeding as above we can also compute the conditional probabilites for $A_{n}$ and $B_{m} \circ \theta_{v}$ separately and deduce

$$
\begin{aligned}
\mathbf{P}_{0}\left(A_{n}, B_{m}\right. & \left.\circ \theta_{v} \mid I_{T}=a, H_{I}=s\right) \\
& =\mathbf{P}_{0}\left(A_{n} \mid I_{T}=a, H_{I}=s\right) \mathbf{P}_{0}\left(B_{m} \circ \theta_{v} \mid I_{T}=a, H_{I}=s\right) .
\end{aligned}
$$

As is seen from (3.12) the quantity

$$
\mathbf{P}_{0}\left(B_{m} \circ \theta_{v} \mid I_{T}=a, H_{I}=s\right)
$$

is a function of the difference $v-s$ only, and we find the desired description of the post-process by letting $v \downarrow s$ (and applying the Lebesgue dominated convergence theorem). To remove the conditioning with respect to $H_{I}$ in (3.13) observe from (3.9) that

$$
\mathbf{P}_{0}\left(H_{I} \in d s \mid I_{T}=a\right)=\frac{\widehat{\eta}_{0}(a, s)}{\mathrm{e}^{a \sqrt{2 \lambda}}} d s
$$

and, hence,

$$
\begin{aligned}
\mathbf{P}_{0}\left(A_{n} \mid I_{T}\right. & =a)=\widehat{p}^{+}\left(u_{1} ; 0, x_{1}\right) 2 d x_{1} \\
& \times \cdots \widehat{p}^{+}\left(u_{n}-u_{n-1} ; x_{n-1}, x_{n}\right) 2 d x_{n} \frac{\mathrm{e}^{-\left(x_{n}-a\right) \sqrt{2 \lambda}}}{\mathrm{e}^{-a \sqrt{2 \lambda}}},
\end{aligned}
$$

which means that the pre-process is as stated, and, moreover, the claimed conditional independence holds. 
It is possible to prove claim 1 also via direct computations with finite dimensional distributions; however, we do not present this proof since, as seen below, the result is in the core of the approach with excursions.

c) Excursion theoretical proof. The excursion process associated with the excursions above the running minimum is defined similarly as the corresponding process with running maximum in Section 2. Indeed, let for $a<0$

$$
H_{a-}:=\inf \left\{t: X_{t}<a\right\},
$$

and, if $H_{a-}>H_{a}$,

$$
\Xi_{a}^{-}(u):=X_{u+H_{a}}-a, \quad 0 \leq u<H_{a-}-H_{a} .
$$

Then the process

$$
\Xi^{-}=\left\{\Xi_{a}^{-}: a \leq 0\right\}
$$

is a homogeneous Poisson point process, and is called the excursion process for excursions above the running minimum. We remark that $\Xi^{-}$is identical in law with the excursion process for excursions from 0 of a reflecting Brownian motion. The Ito excursion measure associated with $\Xi^{-}$is denoted by $n^{-}$(for different descriptions of $n^{-}$, see Revuz and Yor [12]).

Let $F_{1}$ and $F_{2}$ be measurable mappings from $\mathcal{C}\left(\mathbf{R}_{+}, \mathbf{R}\right)$ to $\mathbf{R}_{+}$. Now we can write

$$
\begin{aligned}
\Delta:= & \mathbf{E}\left(F_{1}\left(X_{u}: u \leq H_{I}\right) F_{2}\left(X_{H_{I}+u}-I_{T}: u \leq T-H_{I}\right)\right) \\
& =\mathbf{E}\left(\sum_{a<0} F_{1}\left(X_{u}: u \leq H_{a}\right) F_{2}\left(\Xi_{a}^{-}(u): u \leq T-H_{a}\right) \mathbf{1}_{\left\{H_{a} \leq T<H_{a-}\right\}}\right),
\end{aligned}
$$

where the sum is over all points of $\Xi^{-}$(but simplifies, for every $\omega$ a.s., only to one term). Let $\mathcal{E}$ denote the excursion space and $\varepsilon$ a generic excursion. By the compensation formula for Poisson point processes (see Bertoin [2])

$$
\begin{aligned}
& \Delta=\int_{-\infty}^{0} d a \mathbf{E}_{0}\left(F_{1}\left(X_{u}: u \leq H_{a}\right) \mathbf{1}_{\left\{H_{a}<T\right\}}\right. \\
&\left.\times \int_{\mathcal{E}} F_{2}\left(\varepsilon_{u}: u \leq T-H_{a}\right) \mathbf{1}_{\left\{T-H_{a}<\zeta\right\}}(\varepsilon) n^{-}(d \varepsilon)\right) \\
&=\int_{-\infty}^{0} d a \mathbf{E}_{0}\left(F_{1}\left(X_{u}: u \leq H_{a}\right) \mathrm{e}^{-\lambda H_{a}}\right) \\
& \\
& \times \int_{\mathcal{E}} n^{-}(d \varepsilon) \mathbf{E}_{0}\left(F_{2}\left(\varepsilon_{u}: u \leq T\right) \mathbf{1}_{\{T<\zeta\}}(\varepsilon)\right)
\end{aligned}
$$


where the notation $\zeta(\varepsilon)$ is for the life time of $\varepsilon$ and in the second step the fact that $T$ is an exponentially distributed random variable independent of $X$ is used. Notice that (3.14) yields, when choosing $F_{1} \equiv 1$,

$$
\begin{aligned}
& \mathbf{E}_{0}\left(F_{2}\left(X_{H_{I}+u}-I_{T}: u \leq T-H_{I}\right)\right) \\
& =\frac{1}{\sqrt{2 \lambda}} \int_{\mathcal{E}} n^{-}(d \varepsilon) \mathbf{E}_{0}\left(F_{2}\left(\varepsilon_{u}: u \leq T\right) \mathbf{1}_{\{T<\zeta\}}(\varepsilon)\right) .
\end{aligned}
$$

By absolute continuity,

$$
\mathbf{E}_{0}\left(F_{1}\left(X_{u}: u \leq H_{a}\right) \mathrm{e}^{-\lambda H_{a}}\right)=\mathbf{E}_{0}^{-\sqrt{2 \lambda}}\left(F_{1}\left(X_{u}: u \leq H_{a}\right)\right) \mathrm{e}^{a \sqrt{2 \lambda}}
$$

and, since $-I_{T}$ is exponentially distributed with parameter $\sqrt{2 \lambda}$, we have

$$
\begin{aligned}
\Delta=\int_{-\infty}^{0} d a & \mathbf{E}_{0}^{-\sqrt{2 \lambda}}\left(F_{1}\left(X_{u}: u \leq H_{a}\right)\right) \mathbf{P}\left(I_{T} \in d a\right) \\
& \times \frac{1}{\sqrt{2 \lambda}} \int_{\mathcal{E}} n^{-}(d \varepsilon) \mathbf{E}_{0}\left(F_{2}\left(\varepsilon_{u}: u \leq T\right) \mathbf{1}_{\{T<\zeta\}}(\varepsilon)\right) .
\end{aligned}
$$

Consequently, the processes $\left\{X_{u}: u \leq H_{I}\right\}$ and $\left\{X_{H_{I}+u}-I_{T}: u \leq T-H_{I}\right\}$ are independent and, hence, also $\left\{X_{u}: u \leq H_{I}\right\}$ and $\left\{X_{T-t}-X_{T}: u \leq\right.$ $\left.T-H_{I}\right\}$ are independent. Moreover, $\left\{X_{u}: u \leq H_{I}\right\}$ given $I_{T}=a$ is identical in law with $\operatorname{BM}(-\sqrt{2 \lambda})$ killed at the first hitting time of $a$. To prove that $\left\{X_{T-t}-X_{T}: u \leq T-H_{I}\right\}$ given $X_{T}-I_{T}=b$ is identical in law with $\operatorname{BM}(-\sqrt{2 \lambda})$ killed at the first hitting time of $-b$ observe first that

$$
\begin{aligned}
& \int_{\mathcal{E}} n^{-}(d \varepsilon) \mathbf{E}_{0}\left(F_{2}\left(\varepsilon_{u}: u \leq T\right) \mathbf{1}_{\{T<\zeta\}}(\varepsilon)\right) \\
&=\lambda \int_{0}^{\infty} d t \mathrm{e}^{-\lambda t} n^{-}\left(F_{2}\left(\varepsilon_{u}: u \leq t\right) \mathbf{1}_{\{t<\zeta\}}(\varepsilon)\right) .
\end{aligned}
$$

Next we claim that

$$
\begin{aligned}
\int_{0}^{\infty} d t \mathrm{e}^{-\lambda t} n^{-} & \left(F_{2}\left(\varepsilon_{u}: u \leq t\right) \mathbf{1}_{\{t<\zeta\}}(\varepsilon)\right) \\
& =2 \int_{0}^{\infty} d b \mathbf{E}_{b}\left(\mathrm{e}^{-\lambda H_{0}} F_{2}\left(X_{H_{0}-u}: u \leq H_{0}\right)\right) .
\end{aligned}
$$

Indeed, (3.16) for $\lambda=0$ is formula 5 in Biane and Yor [3] Théorème $6.1 \mathrm{p}$. 79 and the validity for $\lambda>0$ is easily verified by inspecting the proof in [3] 
p. 79. Hence, by spatial symmetry,

$$
\begin{aligned}
& \mathbf{E}_{0}\left(F_{2}\left(X_{H_{I}+u}-I_{T}: u \leq T-H_{I}\right)\right) \\
& =\sqrt{2 \lambda} \int_{0}^{\infty} d b \mathbf{E}_{b}\left(\mathrm{e}^{-\lambda H_{0}} F_{2}\left(X_{H_{0}-u}: u \leq H_{0}\right)\right) \\
& =\sqrt{2 \lambda} \int_{0}^{\infty} d b \mathbf{E}_{0}\left(\mathrm{e}^{-\lambda H_{-b}} F_{2}\left(b+X_{H_{-b}-u}: u \leq H_{-b}\right)\right) .
\end{aligned}
$$

Reversing here time and using absolute continuity yield

$$
\begin{aligned}
\mathbf{E}_{0}\left(F _ { 2 } \left(X_{T}\right.\right. & \left.\left.-X_{T-u}: u \leq T-H_{I}\right)\right) \\
& =\sqrt{2 \lambda} \int_{0}^{\infty} d b \mathbf{E}_{0}\left(\mathrm{e}^{-\lambda H_{b}} F_{2}\left(X_{s}: s \leq H_{b}\right)\right) \\
& =\sqrt{2 \lambda} \int_{0}^{\infty} d b \mathbf{E}_{0}^{\sqrt{2 \lambda}}\left(F_{2}\left(X_{s}: s \leq H_{b}\right)\right) \mathrm{e}^{-b \sqrt{2 \lambda}}
\end{aligned}
$$

which proves the first claim of the theorem.

It remains to verify claim 2 (iii). For a fixed $t>0$ we obtain from (3.17)

$$
\begin{aligned}
\widehat{\Delta} & :=\mathbf{E}_{0}\left(F_{2}\left(X_{H_{I}+u}-I_{T}: u \leq t\right) \mathbf{1}_{\left\{t \leq T-H_{I}\right\}}\right) \\
& =\sqrt{2 \lambda} \int_{0}^{\infty} d b \mathbf{E}_{b}\left(\mathrm{e}^{-\lambda H_{0}} F_{2}\left(X_{H_{0}-u}: u \leq t\right) \mathbf{1}_{\left\{t \leq H_{0}\right\}}\right) .
\end{aligned}
$$

According to Williams' time reversal theorem the process $\left\{X_{H_{0}-u}: 0 \leq u<\right.$ $H_{0}$ \} under $\mathbf{P}_{b}$ is identical in law with $\operatorname{BES}(3)$ started from 0 and killed at the last exit time at $b$. Consequently, letting $\gamma_{b}$ denote the last exit time we have

$$
\begin{aligned}
\widehat{\Delta} & =\sqrt{2 \lambda} \int_{0}^{\infty} d b \mathbf{Q}_{0}\left(\mathrm{e}^{-\lambda \gamma_{b}} F_{2}\left(X_{u}: u \leq t\right) \mathbf{1}_{\left\{t \leq \gamma_{b}\right\}}\right) \\
& =\sqrt{2 \lambda} \mathrm{e}^{-\lambda t} \int_{0}^{\infty} d b \mathbf{Q}_{0}\left(F_{2}\left(X_{u}: u \leq t\right) \mathbf{Q}_{X_{t}}\left(\mathrm{e}^{-\lambda \gamma_{b}} \mathbf{1}_{\left\{\gamma_{b}>0\right\}}\right)\right)
\end{aligned}
$$

by the Markov property. The distribution of $\gamma_{b}$ is well known (see Pitman and Yor [10]) and it holds

$$
\mathbf{Q}_{r}\left(\mathrm{e}^{-\lambda \gamma_{b}} \mathbf{1}_{\left\{\gamma_{b}>0\right\}}\right)=\frac{1}{r \sqrt{2 \lambda}} \operatorname{sh}((b \wedge r) \sqrt{2 \lambda}) \mathrm{e}^{-(b \vee r) \sqrt{2 \lambda}} .
$$


We have now

$$
\begin{aligned}
\widehat{\Delta} & =\sqrt{2 \lambda} \mathrm{e}^{-\lambda t} \mathbf{Q}_{0}\left(F_{2}\left(X_{u}: u \leq t\right) \int_{0}^{\infty} d b \mathbf{Q}_{X_{t}}\left(\mathrm{e}^{-\lambda \gamma_{b}} \mathbf{1}_{\left\{\gamma_{b}>0\right\}}\right)\right) \\
& =\mathrm{e}^{-\lambda t} \mathbf{Q}_{0}\left(F_{2}\left(X_{u}: u \leq t\right) \frac{1-\mathrm{e}^{-X_{t} \sqrt{2 \lambda}}}{X_{t} \sqrt{2 \lambda}}\right) \\
& =\mathbf{Q}_{0}\left(F_{2}\left(X_{u}: u \leq t\right) \frac{h_{3}\left(X_{t}\right)}{X_{t} \sqrt{2 \lambda}} \mathbf{1}_{\{t \leq T\}}\right),
\end{aligned}
$$

where $h_{3}$ is as in (3.2). Define $h_{4}(x):=h_{3}(x) / x$ for $x>0$ and $h_{4}(0):=\sqrt{2 \lambda}$, and notice that $h_{4}$ is right continuous at 0 . Consequently,

$$
\begin{aligned}
\mathbf{E}_{0}\left(F _ { 2 } \left(X_{H_{I}+u}-\right.\right. & \left.\left.I_{T}: u \leq t\right) \mathbf{1}_{\left\{t \leq T-H_{I}\right\}}\right) \\
& =\mathbf{Q}_{0}\left(F_{2}\left(X_{u}: u \leq t\right) \frac{h_{4}\left(X_{t}\right)}{h_{4}(0)} \mathbf{1}_{\{t \leq T\}}\right) .
\end{aligned}
$$

It is easy to verify that for $x>0$

$$
\mathcal{G}^{R} h_{4}(x)-\lambda h_{4}(x)=-\frac{\lambda}{x}<0,
$$

where $\mathcal{G}^{R}$ is the infinitesimal generator of $\operatorname{BES}(3)$, see (2.6). Using Itô's formula it is seen that $h_{4}$ is $\lambda$-excessive for $\operatorname{BES}(3)$. Consequently, the post $H_{I}$-process is identical in law with the Doob $h$-transform with $h=h_{4}$ of exponentially killed $\operatorname{BES}(3)$. The generator of this transform can be computed in the usual way, and is seen to coincide when $a=0$ with $\mathcal{G}^{Z}$ given in (3.1).

Remark 3.3. 1. Informally, given $I_{T}=a$ the post- $H_{I}$-process is identical in law with a Brownian motion killed at time $\zeta:=T \wedge H_{a}$ and conditioned by the event $X_{\zeta-}>a$. Let $\mathbf{P}^{Z}$ denote the probability measure associated with the diffusion $Z$ introduced in Theorem 3.2. Then for any finite stopping time $U$ and $\Lambda_{U} \in \mathcal{F}_{U}$ it holds

$$
\begin{aligned}
\mathbf{P}_{x}^{Z}\left(\Lambda_{U}\right) & =\frac{1}{h_{3}(x-a)} \mathbf{E}_{x}\left(h_{3}\left(X_{U}-a\right) ; \Lambda_{U}, U<T \wedge H_{a}\right) \\
& =\mathbf{P}_{x}\left(\Lambda_{U}, U<T \wedge H_{a} \mid X_{\zeta-}>a\right) .
\end{aligned}
$$

The diffusion $Z$ can alternatively, as is seen at the end of the above proof, be described as the Doob $h$-transform with $h=h_{4}$ of exponentially killed $\operatorname{BES}(3)$. 
2. From Theorem 3.2 it is clear that $\left\{X_{t}: 0 \leq t<H_{I}\right\}$ and $\left\{X_{H_{I}+t}-I_{T}\right.$ : $\left.0 \leq t<T-H_{I}\right\}$ are independent. In particular, $X_{T}-I_{T}$ and $I_{T}$ are independent. This last property is also easily verified by analyzing the joint distribution of $X_{T}$ and $I_{T}$

$$
\mathbf{P}_{0}\left(I_{T} \in d a, X_{T} \in d z\right)=2 \lambda \mathrm{e}^{2 a \sqrt{2 \lambda}} \mathrm{e}^{-z \sqrt{2 \lambda}} d a d z, \quad a<0, a<z,
$$

which is obtained from (3.8) by integrating with respect to s. Moreover, $X_{T}-I_{T}$ and $-I_{T}$ are seen to be identically distributed the common distribution being the exponential distribution with parameter $\sqrt{2 \lambda}$ (cf. also (3.20) below).

3. Notice also the fact that $H_{I}$ and $T-H_{I}$ are independent and identically $\operatorname{Gamma}(1 / 2, \lambda)$-distributed, i.e.,

$$
\mathbf{P}_{0}\left(H_{I} \in d u\right)=\mathbf{P}_{0}\left(T-H_{I} \in d u\right)=\frac{\sqrt{\lambda}}{\sqrt{\pi u}} \mathrm{e}^{-\lambda u} d u, \quad u>0 .
$$

Next we recall the formulas (see [4] p. 173)

$$
\mathbf{P}_{0}\left(I_{T} \in d a, S_{T} \in d b\right)=\lambda \frac{\operatorname{ch}\left(\frac{1}{2}(b+a) \sqrt{2 \lambda}\right)}{\operatorname{ch}^{3}\left(\frac{1}{2}(b-a) \sqrt{2 \lambda}\right)} d a d b, \quad a<0<b,
$$

and

$$
\mathbf{P}_{0}\left(I_{T} \in d a\right)=\sqrt{2 \lambda} \mathrm{e}^{a \sqrt{2 \lambda}} d a \quad a<0 .
$$

We need also the following result derived as a corollary to Theorem 3.2 but which can also be deduced from the formula 1.1.28.2 p. 191 in [4].

Corollary 3.4. For $a<0<b$

$$
\begin{aligned}
\mathbf{P}_{0}\left(H_{I}<H_{S}, I_{T}\right. & \left.\in d a, S_{T} \in d b\right) \\
& =2 \lambda \frac{(\operatorname{ch}((b-a) \sqrt{2 \lambda})-1) \operatorname{sh}(b \sqrt{2 \lambda})}{\operatorname{sh}^{3}((b-a) \sqrt{2 \lambda})} d a d b .
\end{aligned}
$$

Proof. The conditional independence stated in Theorem 3.2 yields

$$
\begin{aligned}
\mathbf{P}_{0}\left(H_{I}<H_{S}, S_{T}\right. & \left.\in d b \mid I_{T}=a\right) \\
& =\mathbf{P}_{0}\left(H_{I}<H_{S}, S_{H_{I}, T} \in d b \mid I_{T}=a\right) \\
& =\mathbf{P}_{0}\left(S_{H_{I}, T} \in d b \mid I_{T}=a\right) \mathbf{P}_{0}\left(S_{H_{I}}<b \mid I_{T}=a\right),
\end{aligned}
$$


where

$$
S_{H_{I}, T}:=\sup \left\{X_{t}: H_{I} \leq t \leq T\right\} .
$$

From the description of the pre- $H_{I}$ process we have using the well known formula, see e.g. 4] p. 309,

$$
\begin{aligned}
\mathbf{P}_{0}\left(S_{H_{I}}<b \mid I_{T}=a\right) & =\mathbf{P}_{0}^{-\sqrt{2 \lambda}}\left(H_{a}<H_{b}\right) \\
& =\mathrm{e}^{-a \sqrt{2 \lambda}} \frac{\operatorname{sh}(b \sqrt{2 \lambda})}{\operatorname{sh}((b-a) \sqrt{2 \lambda})} .
\end{aligned}
$$

Using the $h$-transform description of $Z$ (cf. Remark 3.3)

$$
\begin{aligned}
\mathbf{P}_{0}\left(S_{H_{I}, T}>b \mid I_{T}=a\right) & =\mathbf{P}_{a}^{Z}\left(H_{b}<\infty\right) \\
& =\lim _{x \downarrow a} \frac{h_{3}(b-a)}{h_{3}(x-a)} \mathbf{P}_{x}\left(H_{b}<H_{a}, H_{b}<T\right) \\
& =\lim _{x \downarrow a} \frac{h_{3}(b-a)}{h_{3}(x-a)} \frac{\operatorname{sh}((x-a) \sqrt{2 \lambda})}{\operatorname{sh}((b-a) \sqrt{2 \lambda})} \\
& =\frac{1-\mathrm{e}^{-(b-a) \sqrt{2 \lambda}}}{\operatorname{sh}((b-a) \sqrt{2 \lambda})} .
\end{aligned}
$$

The formula (3.21) is now obtained from (3.22) when multiplying with the density of $I_{T}$ given in (3.20).

We proceed by refining the path decomposition presented in Theorem 3.2 Recall that $\mathrm{Q}_{x}^{\mu}$ with $\mu, x \geq 0$ denotes the measure under which the coordinate process $X=\left\{X_{t}: t \geq 0\right\}$ is a $\operatorname{BES}(3, \mu)$ started from $x$ (see (2.5) for the generator).

Theorem 3.5. Conditionally on $H_{I}<H_{S}, I_{T}=a, S_{T}=b$ it holds that

1. the pre- $H_{I}$-process $\left\{X_{t}: 0 \leq t \leq H_{I}\right\}$ is identical in law with $\left\{b-X_{t}\right.$ : $\left.0 \leq t \leq H_{b-a}\right\}$ under $\mathbf{Q}_{b}^{\sqrt{2 \lambda}}$.

2. the intermediate process $\left\{X_{H_{I}+t}: 0 \leq t \leq H_{S}-H_{I}\right\}$ is identical in law with $\left\{a+X_{t}: 0 \leq t \leq H_{b-a}\right\}$ under $\mathbf{Q}_{0}^{\sqrt{2 \lambda}}$.

3. the post- $H_{S}$-process $\left\{X_{H_{S}+t}: 0 \leq t \leq T-H_{S}\right\}$ is identical in law with a diffusion $Z^{\circ}$ started from $b$ and having the generator

$$
\mathcal{G}^{Z^{\circ}} u(x)=\frac{1}{2} u^{\prime \prime}(x)+\frac{g^{\prime}(x)}{g(x)} u^{\prime}(x)-\frac{\lambda g(x)-\frac{1}{2} g^{\prime \prime}(x)}{g(x)} u(x),
$$


where $a<x<b$ and

$$
g(x)=\operatorname{sh}((b-a) \sqrt{2 \lambda})-\operatorname{sh}((b-x) \sqrt{2 \lambda})-\operatorname{sh}((x-a) \sqrt{2 \lambda}),
$$

4. the pre- $H_{I}$-process, the intermediate process and the post- $H_{S}$-process are independent.

Proof. From Theorem 3.2, using symmetry of BM, it is seen that conditionally on $S_{T}=b$

P1. the processes $\left\{X_{t}: 0 \leq t \leq H_{S}\right\}$ and $\left\{X_{H_{S}+t}: 0 \leq t \leq T-H_{S}\right\}$ are independent,

P2. the pre- $H_{S^{-}}$-process $\left\{X_{t}: 0 \leq t \leq H_{S}\right\}$ is distributed as $\operatorname{BM}(\sqrt{2 \lambda})$ started from 0 and killed when it reaches $b$,

P3. the post- $H_{S}$-process $\left\{X_{H_{S}+t}: 0 \leq t \leq T-H_{S}\right\}$ is a diffusion $Z^{\downarrow}$ started from $b$ and having the generator

$$
\mathcal{G}^{Z^{\downarrow}} u(x)=\frac{1}{2} u^{\prime \prime}(x)+\frac{h^{\prime}(b-x)}{h(b-x)} u^{\prime}(x)-\frac{\lambda}{h(b-x)} u(x) .
$$

For the pre- $H_{S}$-process we apply the path decomposition theorem of Brownian motion with drift as given in Tanré and Vallois [13] Proposition 3.2. To recall this, let

$$
I_{H_{b}}:=\inf \left\{X_{t}: 0 \leq t \leq H_{b}\right\} \quad \text { and } \quad \widehat{H}_{b}:=\inf \left\{t: X_{t}=I_{H_{b}}\right\} .
$$

Then, under $\mathbf{P}_{0}^{\mu}$, conditionally on $I_{H_{b}}=a$

P4. the processes $\left\{X_{t}: 0 \leq t \leq \widehat{H}_{b}\right\}$ and $\left\{X_{\widehat{H}_{b}+t}: 0 \leq t \leq H_{b}-\widehat{H}_{b}\right\}$ are independent,

P5. the pre- $\widehat{H}_{b}$-process $\left\{X_{t}: 0 \leq t \leq \widehat{H}_{b}\right\}$ is identical in law with $\mathrm{BM}(-\mu)$ started from 0 , conditioned to hit $a$ before $b$, and killed when it hits $a$.

P6. the post- $\widehat{H}_{b}$-process $\left\{X_{\widehat{H}_{b}+t}: 0 \leq t \leq H_{b}-\widehat{H}_{b}\right\}$ is identical in law with $\left\{a+X_{t}: 0 \leq t \leq \widehat{H}_{b-a}\right\}$ under $\mathbf{Q}_{0}^{\mu}$. 
Let $F_{1}, F_{2}$, and $F_{3}$ be measurable mappings from $\mathcal{C}\left(\mathbf{R}_{+}, \mathbf{R}\right)$ to $\mathbf{R}_{+}$, and introduce

$$
F_{1}:=F_{1}\left(\left\{X_{t}: 0 \leq t \leq H_{I}\right\}\right), \quad F_{2}:=F_{2}\left(\left\{X_{H_{I}+t}: 0 \leq t \leq H_{S}-H_{I}\right\}\right),
$$

and

$$
F_{3}:=F_{3}\left(\left\{X_{H_{S}+t}: 0 \leq t \leq T-H_{S}\right\}\right),
$$

where it is assumed that $H_{I}<H_{S}$. Notice that

$$
H_{I} \leq H_{S} \quad \Leftrightarrow \quad I_{H_{S}, T}:=\inf \left\{X_{u}: H_{S} \leq u \leq T\right\}>I_{H_{S}} .
$$

and

$$
I_{H_{S}, T}>I_{H_{S}} \quad \Leftrightarrow \quad I_{H_{S}}=I_{T}
$$

Consider now

$$
\begin{aligned}
\mathbf{E}_{0}\left(F_{1} F_{2} F_{3}\right. & \left.; I_{T} \in d a, H_{I}<H_{S} \mid S_{T}=b\right) \\
& =\mathbf{E}_{0}\left(F_{1} F_{2} F_{3} ; I_{H_{S}} \in d a, I_{H_{S}, T}>a \mid S_{T}=b\right) \\
& =\mathbf{E}_{0}\left(F_{1} F_{2} ; I_{H_{S}} \in d a \mid S_{T}=b\right) \mathbf{E}_{0}\left(F_{3} ; I_{H_{S}, T}>a \mid S_{T}=b\right),
\end{aligned}
$$

where in the second step the conditional independence (see P1) is applied. The first term on the right hand side can be analyzed via properties P4, P5 and P6. In particular, claims 1 and 2 of Theorem 3.5 follow with the help of Lemma 2.3. Moreover, P1 and P4 yield claim 4.

Next we prove claim 3. For this let $\mathbf{P}_{x}^{\downarrow}$ denote the measure in the canonical setting associated with $Z^{\downarrow}$ when started from $x<b$. Hence,

$$
\begin{gathered}
\mathbf{E}_{0}\left(F_{3}\left(\left\{X_{H_{S}+u}: 0 \leq u \leq t\right\}\right) 1_{\left\{t \leq T-H_{S}\right\}} \mid I_{H_{S}, T}>a, S_{T}=b\right) \\
=\mathbf{E}_{b}^{\downarrow}\left(F_{3}\left(\left\{X_{u}: 0 \leq u \leq t\right\}\right) \mathbf{1}_{\{t \leq \zeta\}} \mid I_{\zeta}>a\right),
\end{gathered}
$$

where $\zeta$ denotes the life time and $I_{\zeta}$ the global infimum. By the Markov property,

$$
\begin{aligned}
& \mathbf{E}_{b}^{\downarrow}\left(F_{3}\left(\left\{X_{u}: 0 \leq u \leq t\right\}\right) ; t \leq \zeta, I_{\zeta}>a\right) \\
& \quad=\mathbf{E}_{b}^{\downarrow}\left(F_{3}\left(\left\{X_{u}: 0 \leq u \leq t\right\}\right) \mathbf{1}_{\left\{t<H_{a}\right\}} \mathbf{P}_{X_{t}}^{\downarrow}\left(I_{\zeta}>a\right)\right) .
\end{aligned}
$$

Clearly, for $x \leq b$

$$
\mathbf{P}_{x}^{\downarrow}\left(I_{\zeta}>a\right)=\mathbf{P}_{x}^{\downarrow}\left(H_{a}=\infty\right)=1-\mathbf{P}_{x}^{\downarrow}\left(H_{a}<\infty\right),
$$


and computing as in (3.23) we obtain

$$
\mathbf{P}_{x}^{\downarrow}\left(H_{a}<\infty\right)=\frac{h_{3}(b-a)}{h_{3}(b-x)} \frac{\operatorname{sh}((b-x) \sqrt{2 \lambda})}{\operatorname{sh}((b-a) \sqrt{2 \lambda})}
$$

with $h_{3}$ as in (3.2). Consequently, after some elementary manipulations, for $x \in(a, b)$

$$
\mathbf{P}_{x}^{\downarrow}\left(I_{\zeta}>a\right)=h_{5}(x):=\frac{g(x)}{h_{3}(b-x) \operatorname{sh}((b-a) \sqrt{2 \lambda})}
$$

with $g$ as in (3.24). Since $Z^{\downarrow}$ is the Doob $h$-transform with $h=h_{3}(b-\cdot)$ of BM killed at time $T \wedge H_{b}$ it follows that

$$
\begin{aligned}
\mathbf{E}_{b}^{\downarrow}( & \left.F_{3}\left(\left\{X_{u}: 0 \leq u \leq t\right\}\right) ; t \leq \zeta \mid I_{\zeta}>a\right) \\
& =\mathbf{E}_{b}^{\downarrow}\left(\frac{h_{5}\left(X_{t}\right)}{h_{5}(b)} F_{3}\left(\left\{X_{u}: 0 \leq u \leq t\right\}\right) ; t \leq \zeta\right) \\
& =\lim _{x \uparrow b} \mathbf{E}_{x}\left(\frac{h_{3}\left(b-X_{t}\right) h_{5}\left(X_{t}\right)}{h_{3}(b-x) h_{5}(x)} F_{3}\left(\left\{X_{u}: 0 \leq u \leq t\right\}\right) ; t \leq T \wedge H_{b} \wedge H_{a}\right) \\
& =\lim _{x \uparrow b} \mathbf{E}_{x}\left(\frac{g\left(X_{t}\right)}{g(x)} F_{3}\left(\left\{X_{u}: 0 \leq u \leq t\right\}\right) ; t \leq T \wedge H_{b} \wedge H_{a}\right),
\end{aligned}
$$

and this yields the description 3 of the post process.

Remark 3.6. 1. Informally, the post- $H_{S}$ process is identical in law with a Brownian motion killed at time $\zeta:=T \wedge H_{a} \wedge H_{b}$ and conditioned by the event $X_{\zeta-} \in(a, b)$. Indeed, from [4] 3.0.1 p. 212

$$
\begin{aligned}
\mathbf{P}_{x}\left(X_{\zeta} \in(a, b)\right) & =\mathbf{P}_{x}\left(T<H_{a} \wedge H_{b}\right) \\
& =\mathbf{E}_{x}\left(1-\exp \left(-\lambda H_{a} \wedge H_{b}\right)\right) \\
& =1-\frac{\operatorname{sh}((b-x) \sqrt{2 \lambda})-\operatorname{sh}((x-a) \sqrt{2 \lambda})}{\operatorname{sh}((b-a) \sqrt{2 \lambda})} \\
& =\frac{g(x)}{\operatorname{sh}((b-a) \sqrt{2 \lambda})} .
\end{aligned}
$$

2. It can be proved that the process $\left\{X_{T-t}: 0 \leq t \leq T-H_{S}\right\}$, i.e., the time reversal of the post- $H_{S}$-process is identical in law with $\left\{a+X_{t}: 0 \leq t \leq\right.$ $\left.H_{b-a}\right\}$ under $\mathbf{Q}_{b-a-\xi}^{\sqrt{2 \lambda}}$ with $\xi$ a random variable independent of $X$ having the density

$$
f_{\xi}(x)=\sqrt{2 \lambda} \mathrm{e}^{-x \sqrt{2 \lambda}} /\left(1-\mathrm{e}^{-(b-a) \sqrt{2 \lambda}}\right), \quad 0<x<b-a .
$$


Consequnetly, for $0<x<b-a$

$$
\mathbf{P}\left(S_{T}-X_{T} \in d x \mid S_{T}=b, I_{T}=a, H_{I}<H_{S}\right)=f_{\xi}(x) d x .
$$

\section{Maximum increase and decrease}

In this section we apply the results from the previous sections to find an expression for the joint distribution of the maximum increase and the maximum decrease of Brownian motion up to an independent exponential time $T$. As stated in the Introduction the law of $\left(D_{t}^{+}, D_{t}^{-}\right)$under $P_{x}$ does not depend on $x$ and, hence, we write in the sequel $\mathbf{P}$ instead of $\mathbf{P}_{x}$.

For $s<t$ define the maximum increase on the interval $(s, t)$ via

$$
D_{s, t}^{+}:=\sup _{s \leq u \leq v \leq t}\left(X_{v}-X_{u}\right)
$$

and, analogously, the maximum decrease

$$
D_{s, t}^{-}:=\sup _{s \leq u \leq v \leq t}\left(X_{u}-X_{v}\right) .
$$

With these new notations we have $D_{T}^{-}=D_{0, T}^{-}$and $D_{T}^{+}=D_{0, T}^{+}$.

The path decomposition given in Theorem 3.5 leads us to the following

Proposition 4.1. 1. For $-a<d<b-a$

$$
\begin{aligned}
\mathbf{P}\left(D_{H_{I}}^{-}<d \mid H_{I}<H_{S}\right. & \left.I_{T}=a, S_{T}=b\right) \\
= & \frac{S^{\nu}(a+d)\left(S^{\nu}(b)-S^{\nu}(a)\right)}{S^{\nu}(b)\left(S^{\nu}(a+d)-S^{\nu}(a)\right)} \\
= & \frac{\operatorname{sh}((a+d) \sqrt{2 \lambda}) \operatorname{sh}((b-a) \sqrt{2 \lambda})}{\operatorname{sh}(d \sqrt{2 \lambda}) \operatorname{sh}(b \sqrt{2 \lambda})} \\
= & : f_{1}(d ; a, b),
\end{aligned}
$$

where $S^{\nu}$ is as (2.1) with $\nu=-\sqrt{2 \lambda}$, i.e.,

$$
S^{\nu}(x):=\frac{1}{2 \sqrt{2 \lambda}}\left(\mathrm{e}^{2 \sqrt{2 \lambda} x}-1\right)=\frac{1}{\sqrt{2 \lambda}} \mathrm{e}^{\sqrt{2 \lambda} x} \operatorname{sh}(x \sqrt{2 \lambda}) .
$$


2. For $0<d<b-a$

$$
\begin{aligned}
\mathbf{P}\left(D_{H_{I}, H_{S}}^{-}<d \mid\right. & \left.H_{I}<H_{S}, I_{T}=a, S_{T}=b\right) \\
& =\frac{S^{\nu}(b-a)}{S^{\nu}(d)} \exp \left(-\frac{b-a-d}{S^{\nu}(d)}-2 \sqrt{2 \lambda}(b-a-d)\right) \\
& =\frac{\operatorname{sh}((b-a) \sqrt{2 \lambda})}{\operatorname{sh}(d \sqrt{2 \lambda})} \exp (-(b-a-d) \sqrt{2 \lambda} \operatorname{coth}(d \sqrt{2 \lambda})) \\
& =: f_{2}(d ; a, b)
\end{aligned}
$$

3. For $0<d<b-a$

$$
\begin{aligned}
\mathbf{P}\left(D_{H_{S}, T}^{-}<d \mid H_{I}\right. & \left.<H_{S}, I_{T}=a, S_{T}=b\right) \\
= & \frac{\operatorname{sh}((b-a) \sqrt{2 \lambda})(\operatorname{ch}(d \sqrt{2 \lambda})-1)}{\operatorname{sh}(d \sqrt{2 \lambda})(\operatorname{ch}((b-a) \sqrt{2 \lambda})-1)} \\
= & : f_{3}(d ; a, b)
\end{aligned}
$$

Moreover, conditionally on $I_{T}, S_{T}$ and $H_{I}<H_{S}$ the variables $D_{H_{I}}^{-}, D_{H_{I}, H_{S}}^{-}$, and $D_{H_{S}, T}^{-}$are independent.

Proof. Claim 1. Because

$$
D_{T}^{-}=\sup _{0 \leq v \leq t}\left(\sup _{0 \leq u \leq v} X_{u}-X_{v}\right)
$$

and since, conditionally on $I_{T}=a, H_{I}$ is the first hitting time of $a$ we have

$$
D_{H_{I}}^{-}=\sup _{0 \leq v \leq H_{I}} X_{u}-a
$$

and, hence, for $-a<d<b-a$

$$
\left\{D_{H_{I}}^{-}<d\right\}=\left\{H_{a}<H_{a+d}\right\} .
$$

From Theorem 3.5 and Lemma 2.3 it follows that

$$
\begin{aligned}
\mathbf{P}\left(D_{H_{I}}^{-}<d \mid\right. & \left.H_{I}<H_{S}, I_{T}=a, S_{T}=b\right) \\
& =\mathbf{Q}_{b}^{\sqrt{2 \lambda}}\left(H_{b-a}<H_{b-a-d}\right) \\
& =\mathbf{P}_{b}^{\sqrt{2 \lambda}}\left(H_{b-a}<H_{b-a-d} \mid H_{b-a}<H_{0}\right) .
\end{aligned}
$$


Since $\left\{b-X_{t}: t \geq 0\right\}$ under $\mathbf{P}_{b}^{\sqrt{2 \lambda}}$ is distributed as $\left\{X_{t}: t \geq 0\right\}$ under $\mathbf{P}_{0}^{-\sqrt{2 \lambda}}$, we have

$$
\begin{aligned}
\mathbf{P}\left(D_{H_{I}}^{-}<d \mid\right. & \left.H_{I}<H_{S}, I_{T}=a, S_{T}=b\right) \\
& =\mathbf{P}_{0}^{-\sqrt{2 \lambda}}\left(H_{a}<H_{a+d} \mid H_{a}<H_{b}\right) \\
& =\mathbf{P}_{0}^{-\sqrt{2 \lambda}}\left(H_{a}<H_{a+d}\right) / \mathbf{P}_{0}^{-\sqrt{2 \lambda}}\left(H_{a}<H_{b}\right) \\
& =\frac{S^{\nu}(a+d)-S^{\nu}(0)}{S^{\nu}(a+d)-S^{\nu}(a)} \frac{S^{\nu}(b)-S^{\nu}(a)}{S^{\nu}(b)-S^{\nu}(0)},
\end{aligned}
$$

where the fact that $S^{\nu}$ is the scale function of $\operatorname{BM}(-\sqrt{2 \lambda})$ is used. Observing that for $y>x$

$$
S^{\nu}(y)-S^{\nu}(x)=\frac{1}{\sqrt{2 \lambda}} \mathrm{e}^{\sqrt{2 \lambda}(y+x)} \operatorname{sh}((y-x) \sqrt{2 \lambda})
$$

leads immediately to the claimed formula.

Claim 2. This follows directly from Proposition 2.5] and Theorem 3.5.

Claim 1. Using again Theorem 3.5

$$
\mathbf{P}\left(D_{H_{S}, T}^{-}<d \mid H_{I}<H_{S}, I_{T}=a, S_{T}=b\right)=\mathbf{P}_{b}^{Z^{\circ}}\left(H_{b-d}=+\infty\right) .
$$

By the $h$-transform description of $Z^{\circ}$ (cf. Remark 3.6),

$$
\begin{aligned}
\mathbf{P}_{b}^{Z^{\circ}}\left(H_{b-d}<+\infty\right) & =\lim _{x \uparrow b} \mathbf{P}_{x}^{Z^{\circ}}\left(H_{b-d}<+\infty\right) \\
& =\lim _{x \uparrow b} \frac{1}{g(x)} \mathbf{E}_{x}\left(g\left(X_{H_{b-d}}\right) ; H_{b-d}<T \wedge H_{b} \wedge H_{a}\right) \\
& =\lim _{x \uparrow b} \frac{g(b-d)}{g(x)} \mathbf{E}_{x}\left(\mathrm{e}^{-\lambda H_{b-d}} ; H_{b-d}<H_{b}\right) \\
& =\lim _{x \uparrow b} \frac{g(b-d)}{g(x)} \frac{\operatorname{sh}((b-x) \sqrt{2 \lambda})}{\operatorname{sh}(d \sqrt{2 \lambda})}
\end{aligned}
$$

where $g$ is as in (3.24), i.e., for $a<x<b$

$$
g(x)=\operatorname{sh}((b-a) \sqrt{2 \lambda})-\operatorname{sh}((b-x) \sqrt{2 \lambda})-\operatorname{sh}((x-a) \sqrt{2 \lambda}) .
$$

Straightforward computations yield now the claimed formula. 
Applying symmetry properties of Brownian motion gives us the following formulas

$$
\begin{aligned}
& \mathbf{P}\left(D_{T}^{+}<\alpha, D_{T}^{-}<\beta\right)=\mathbf{P}\left(D_{T}^{+}<\beta, D_{T}^{-}<\alpha\right) \\
& \begin{aligned}
\mathbf{P}\left(D_{T}^{+}<\alpha, D_{T}^{-}\right. & \left.<\beta, H_{S}<H_{I}\right) \\
& =\mathbf{P}\left(D_{T}^{+}<\beta, D_{T}^{-}<\alpha, H_{I}<H_{S}\right)
\end{aligned}
\end{aligned}
$$

and

$$
\begin{aligned}
\mathbf{P}\left(D_{T}^{+}<\alpha, D_{T}^{-}<\beta\right)=\mathbf{P}\left(D_{T}^{+}<\right. & \left.\alpha, D_{T}^{-}<\beta, H_{I}<H_{S}\right) \\
& +\mathbf{P}\left(D_{T}^{+}<\beta, D_{T}^{-}<\alpha, H_{I}<H_{S}\right) .
\end{aligned}
$$

The distribution and the density function of $\left(D_{T}^{+}, D_{T}^{-}\right)$will result as a corollary of the next theorem when applying the formulas (4.2), (4.3), and (4.4).

Theorem 4.2. For $\beta>0$ and $\varphi: \mathbf{R}_{+} \mapsto \mathbf{R}_{+}$bounded and measurable it holds

$$
\begin{gathered}
\mathbf{E}\left(\varphi\left(D_{T}^{+}\right) ; D_{T}^{-}<\beta, H_{I}<H_{S}\right) \\
=\sqrt{2 \lambda} \frac{(\operatorname{ch}(\beta \sqrt{2 \lambda})-1)^{2}}{\operatorname{sh}^{3}(\beta \sqrt{2 \lambda})} \int_{\beta}^{\infty} d x \varphi(x) \exp (-(x-\beta) \sqrt{2 \lambda} \operatorname{coth}(\beta \sqrt{2 \lambda})) \\
\quad+\sqrt{2 \lambda} \int_{0}^{\beta} d x \varphi(x) \frac{(\operatorname{ch}(x \sqrt{2 \lambda})-1)^{2}}{\operatorname{sh}^{3}(x \sqrt{2 \lambda})} .
\end{gathered}
$$

Proof. Notice first that if $H_{I}<H_{S}$ then

$$
D_{T}^{+}=S_{T}-I_{T} \quad \text { and } \quad D_{T}^{-}=D_{H_{I}}^{-} \vee D_{H_{I}, H_{S}}^{-} \vee D_{H_{S}, T}^{-},
$$

and, therefore,

$$
\begin{aligned}
& \Delta:=\mathbf{E}\left(\varphi\left(D_{T}^{+}\right) ; D_{T}^{-}<\beta, H_{I}<H_{S}\right) \\
& \quad=\mathbf{E}\left(\varphi\left(S_{T}-I_{T}\right) ; D_{H_{I}}^{-}<\beta, D_{H_{I}, H_{S}}^{-}<\beta, D_{H_{S}, T}^{-}<\beta, H_{I}<H_{S}\right)
\end{aligned}
$$

Taking the conditional expectation with respect to the $\sigma$-algebra generated by $\left(I_{T}, S_{T}, \mathbf{1}_{\left\{H_{I}<H_{S}\right\}}\right)$ and using Proposition 4.1 we obtain

$$
\begin{array}{rl}
\Delta=\int_{-\infty}^{0} & d a \int_{0}^{+\infty} d b \varphi(b-a) f(a, b) \\
& \times \mathbf{1}_{\{\beta>-a\}}\left(f_{1}(\beta ; a, b) f_{2}(\beta ; a, b) f_{3}(\beta ; a, b) \mathbf{1}_{\{\beta<b-a\}}+\mathbf{1}_{\{\beta>b-a\}}\right)
\end{array}
$$


with $f_{i}, i=1,2,3$, as in Proposition 4.1 and

$$
\begin{aligned}
f(a, b) & =2 \lambda \frac{(\operatorname{ch}((b-a) \sqrt{2 \lambda})-1) \operatorname{sh}(b \sqrt{2 \lambda})}{\operatorname{sh}^{3}((b-a) \sqrt{2 \lambda})} \\
& =\mathbf{P}\left(I_{T} \in d a, S_{T} \in d b, H_{I}<H_{S}\right) / d a d b
\end{aligned}
$$

(cf. Corollary (3.4). Introducing in (4.6) new variables via $x=b-a$ and $y=b$ allows us to write

$$
\Delta=\int_{0}^{\infty} d x \varphi(x)\left(\Delta_{1}(x) \Delta_{2}(x)+\Delta_{3}(x)\right)
$$

where

$$
\begin{gathered}
\Delta_{1}(x):=\frac{2 \lambda(\operatorname{ch}(\beta \sqrt{2 \lambda})-1)}{\operatorname{sh}^{3}(\beta \sqrt{2 \lambda})} \exp (-(x-\beta) \sqrt{2 \lambda} \operatorname{coth}(\beta \sqrt{2 \lambda})), \\
\Delta_{2}(x):=\mathbf{1}_{\{\beta<x\}} \int_{x-\beta}^{x} \operatorname{sh}((y-x+\beta) \sqrt{2 \lambda}) d y=\frac{\operatorname{ch}(\beta \sqrt{2 \lambda})-1}{\sqrt{2 \lambda}} \mathbf{1}_{\{\beta<x\}},
\end{gathered}
$$

and

$$
\begin{aligned}
\Delta_{3}(x) & :=\frac{2 \lambda(\operatorname{ch}(x \sqrt{2 \lambda})-1)}{\operatorname{sh}^{3}(x \sqrt{2 \lambda})} \mathbf{1}_{\{\beta>x\}} \int_{0}^{x} \operatorname{sh}(y \sqrt{2 \lambda}) d y \\
& =\frac{\sqrt{2 \lambda}(\operatorname{ch}(x \sqrt{2 \lambda})-1)^{2}}{\operatorname{sh}^{3}(x \sqrt{2 \lambda})} \mathbf{1}_{\{\beta>x\}} .
\end{aligned}
$$

The claimed formula (4.5) results now easily from (4.7).

Before giving results for the joint distribution of $D_{T}^{+}$and $D_{T}^{-}$we consider the marginal distributions under the condition $H_{I}<H_{S}$.

Corollary 4.3. For $\alpha, \beta>0$

$$
\mathbf{P}\left(D_{T}^{+} \in d \alpha, H_{I}<H_{S}\right)=\frac{\sqrt{2 \lambda}(\operatorname{ch}(\alpha \sqrt{2 \lambda})-1)^{2}}{\operatorname{sh}^{3}(\alpha \sqrt{2 \lambda})} d \alpha,
$$




$$
\begin{gathered}
\mathbf{P}\left(D_{T}^{+}<\alpha, H_{I}<H_{S}\right)=\frac{\operatorname{ch}(\alpha \sqrt{2 \lambda})-1}{2(\operatorname{ch}(\alpha \sqrt{2 \lambda})+1)} \\
\mathbf{P}\left(D_{T}^{-}<\beta, H_{I}<H_{S}\right)=\frac{(\operatorname{ch}(\beta \sqrt{2 \lambda})-1)(\operatorname{ch}(\beta \sqrt{2 \lambda})+2)}{2(\operatorname{ch}(\beta \sqrt{2 \lambda})+1) \operatorname{ch}(\beta \sqrt{2 \lambda})},
\end{gathered}
$$

and

$$
\mathbf{P}\left(D_{T}^{+}<\alpha\right)=1-\frac{1}{\operatorname{ch}(\alpha \sqrt{2 \lambda})}
$$

Proof. Letting $\beta \rightarrow+\infty$ in (4.5) yields (4.8). To compute the distribution function in (4.9) notice that

$$
\frac{(\operatorname{ch}(\alpha \sqrt{2 \lambda})-1)^{2}}{\operatorname{sh}^{3}(\alpha \sqrt{2 \lambda})}=\frac{\operatorname{sh}(\alpha \sqrt{2 \lambda} / 2)}{2 \operatorname{ch}^{3}(\alpha \sqrt{2 \lambda} / 2)}
$$

and hence

$$
\begin{aligned}
\mathbf{P}\left(D_{T}^{+}<\alpha, H_{I}<H_{S}\right) & =\frac{1}{2}\left(1-\frac{1}{\operatorname{ch}^{2}(\alpha \sqrt{2 \lambda} / 2)}\right) \\
& =\frac{1}{2}\left(1-\frac{2}{1+\operatorname{ch}(\alpha \sqrt{2 \lambda})}\right)
\end{aligned}
$$

and (4.9) follows easily. Choosing $\varphi \equiv 1$ in (4.5), integrating therein and using the above computation it is straightforward to derive (4.10). For (4.11) use (4.4), (4.8), and (4.10).

The proof of Theorem 4.2 shows that the way to express the law of $\left(D_{T}^{+}, D_{T}^{-}\right)$in (4.5) is very natural. However, we need also "more standard" representations obtained when choosing in (4.5) $\varphi(x)=\mathbf{1}_{\{x<\alpha\}}$.

Proposition 4.4. The distribution function of $\left(D_{T}^{+}, D_{T}^{-}, \mathbf{1}_{\left\{H_{I}<H_{S}\right\}}\right)$ is given for $\alpha, \beta \geq 0$ by

$$
\begin{aligned}
\mathbf{P}\left(D_{T}^{+}<\alpha, D_{T}^{-}<\beta, H_{I}<H_{S}\right)=\frac{1}{2} \frac{\operatorname{ch}((\alpha \wedge \beta) \sqrt{2 \lambda})-1}{\operatorname{ch}((\alpha \wedge \beta) \sqrt{2 \lambda})+1} \\
+\frac{\operatorname{ch}(\beta \sqrt{2 \lambda})-1}{\operatorname{ch}(\beta \sqrt{2 \lambda})(\operatorname{ch}(\beta \sqrt{2 \lambda})+1)} \\
\quad \times(1-\exp (-(\alpha-\beta) \sqrt{2 \lambda} \operatorname{coth}(\beta \sqrt{2 \lambda}))) \mathbf{1}_{\{\beta<\alpha\}} .
\end{aligned}
$$


Notice that

$$
H_{I}<H_{S} \quad \Leftrightarrow \quad D_{T}^{+} \geq D_{T}^{-},
$$

explaining the appearance of the indicator function $\mathbf{1}_{\{\beta<\alpha\}}$ in (4.12). Moreover, combining (4.12) with (4.2), (4.3), (4.4), and (4.11) yields

Proposition 4.5. The distribution function of $\left(D_{T}^{+}, D_{T}^{-}\right)$is given for $\alpha, \beta>$ 0 by

$$
\mathbf{P}\left(D_{T}^{+}<\alpha, D_{T}^{-}<\beta\right)= \begin{cases}u(\alpha, \beta), & \alpha \leq \beta \\ u(\beta, \alpha), & \alpha \geq \beta\end{cases}
$$

where

$$
u(\alpha, \beta):=1-\frac{1}{\operatorname{ch}(\alpha \sqrt{2 \lambda})}-v(\alpha, \beta)
$$

and

$$
v(\alpha, \beta):=\frac{(\operatorname{ch}(\alpha \sqrt{2 \lambda})-1) \exp (-(\beta-\alpha) \sqrt{2 \lambda} \operatorname{coth}(\alpha \sqrt{2 \lambda}))}{\operatorname{ch}(\alpha \sqrt{2 \lambda})(\operatorname{ch}(\alpha \sqrt{2 \lambda})+1)} .
$$

In particular,

$$
\mathbf{P}\left(D_{T}^{+}>\alpha, D_{T}^{-}<\beta\right)=v(\alpha \wedge \beta, \alpha \vee \beta) \text {. }
$$

We are now able to determine the density function of $\left(D_{T}^{+}, D_{T}^{-}\right)$. Differentiating in (4.14) leads after some calculations to

Proposition 4.6. For $\alpha, \beta>0$

$$
\mathbf{P}\left(D_{T}^{+} \in d \alpha, D_{T}^{-} \in d \beta\right)=f_{+,-}(\alpha \vee \beta, \alpha \wedge \beta) d \alpha d \beta
$$

where with $x>y>0$

$$
\begin{aligned}
& f_{+,-}(x, y):=\frac{2 \lambda}{(\operatorname{ch}(y \sqrt{2 \lambda})+1)^{2}}\left(2+\frac{(x-y) \sqrt{2 \lambda}}{\operatorname{sh}(y \sqrt{2 \lambda})}\right) \\
& \times \exp (-(x-y) \sqrt{2 \lambda} \operatorname{coth}(y \sqrt{2 \lambda})) .
\end{aligned}
$$

Remark 4.7. To increase understanding of the distribution of $\left(D_{T}^{+}, D_{T}^{-}\right)$ notice that

$$
\mathbf{P}\left(D_{T}^{+} \in d \alpha, D_{T}^{-} \in d \beta \mid H_{I}<H_{S}\right)=2 f_{+,-}(\alpha, \beta) \mathbf{1}_{\{\alpha>\beta\}} d \alpha d \beta
$$


Consequently, for $\alpha>0, \beta>0$

$$
\begin{aligned}
& \mathbf{P}\left(D_{T}^{+}-D_{T}^{-} \in d \alpha, D_{T}^{-} \in d \beta \mid H_{I}<H_{S}\right) \\
&= \frac{4 \lambda}{(\operatorname{ch}(\beta \sqrt{2 \lambda})+1)^{2}}\left(2+\frac{\alpha \sqrt{2 \lambda}}{\operatorname{sh}(\beta \sqrt{2 \lambda})}\right) \\
& \quad \times \exp (-\alpha \sqrt{2 \lambda} \operatorname{coth}(\beta \sqrt{2 \lambda})) d \alpha d \beta .
\end{aligned}
$$

Define two new random variables

$$
X:=\sqrt{2 \lambda}\left(D_{T}^{+}-D_{T}^{-}\right) / \operatorname{sh}\left(\sqrt{2 \lambda} D_{T}^{-}\right)
$$

and

$$
Y:=\operatorname{ch}\left(\sqrt{2 \lambda} D_{T}^{-}\right) .
$$

Then for $x>0, y>1$

$$
\mathbf{P}\left(X \in d x, Y \in d y \mid H_{I}<H_{S}\right)=\frac{2(2+x)}{(1+y)^{2}} \mathrm{e}^{-x y} d x d y .
$$

In particular, for $y>1$

$$
\mathbf{P}\left(Y \in d y \mid H_{I}<H_{S}\right)=\frac{2(2 y+1)}{(y(1+y))^{2}} d y
$$

and for $u>1$

$$
\mathbf{P}\left(Y>u \mid H_{I}<H_{S}\right)=\frac{2}{u(1+u)} .
$$

Finally, as an application of Proposition 4.6. we compute the covariance of $D_{t}^{+}$and $D_{t}^{-}$(up to a fixed time $t$ ) and determine therefrom their correlation coefficient.

Corollary 4.8. For all $t \geq 0$

$$
\begin{gathered}
\mathbf{E}\left(D_{t}^{+}\right)=\sqrt{\frac{2}{\pi}} \beta(1) \sqrt{t}=\sqrt{\frac{\pi}{2}} \sqrt{t} \simeq 1.25331 \sqrt{t}, \\
\mathbf{E}\left(\left(D_{t}^{+}\right)^{2}\right)=2 \beta(2) t \simeq 1.83193 t,
\end{gathered}
$$

and

$$
\mathbf{E}\left(D_{t}^{+} D_{t}^{-}\right)=(2 \beta(2)-2 \log 2+1) t \simeq 1.44564 t,
$$

where

$$
\beta(n):=\sum_{k=0}^{\infty}(-1)^{k}(2 k+1)^{-n}, \quad n=1,2, \ldots
$$


Remark 4.9. 1. Recall from Abramowitz and Stegun [1] p. 807, that $\beta(n)$ is called Dirichlet's $\beta$-function. In particular, $\beta(1)=\pi / 2$ and $\beta(2)=0.91596 \ldots$ is Catalan's constant.

2. The variance of $D^{+}$is

$$
\operatorname{Var}\left(D_{t}^{+}\right):=\mathbf{E}\left(\left(D_{t}^{+}\right)^{2}\right)-\left(\mathbf{E}\left(D_{t}^{+}\right)\right)^{2} \simeq 0.26113 t .
$$

The correlation coefficient $\rho$ between $D_{t}^{+}$and $D_{t}^{-}$does not depend on $t$ and is given by

$$
\rho:=\frac{\mathbf{E}\left(D_{t}^{+} D_{t}^{-}\right)-\left(\mathbf{E}\left(D_{t}^{+}\right)\right)^{2}}{\operatorname{Var}\left(D_{t}^{+}\right)} \simeq-0.47936 .
$$

Proof. From the scaling property of BM it follows that

$$
\left(D_{T}^{+}, D_{T}^{-}\right) \stackrel{(\mathrm{d})}{=} \sqrt{T}\left(D_{1}^{+}, D_{1}^{-}\right) .
$$

Since $\mathbf{E}(\sqrt{T})=\sqrt{\pi / \lambda}$ and $T$ is independent of the underlying BM we have using (4.11)

$$
\begin{aligned}
\mathbf{E}\left(D_{1}^{+}\right)=\frac{\mathbf{E}\left(D_{T}^{+}\right)}{\mathbf{E}(\sqrt{T})} & =\sqrt{\frac{\lambda}{\pi}} \int_{0}^{\infty} \mathbf{P}\left(D_{T}^{+}>\alpha\right) d \alpha \\
& =(\sqrt{2 \pi})^{-1} \int_{0}^{\infty} \frac{1}{\operatorname{ch} u} d u=\sqrt{\frac{2}{\pi}} \beta(1)
\end{aligned}
$$

where the series expansion

$$
(\operatorname{ch} u)^{-1}=2 \mathrm{e}^{-u} \sum_{k=0}^{\infty}(-1)^{k} \mathrm{e}^{-2 u k}
$$

is used. This yields the first formula in the statement of the corollary. For the second formula we compute similarly:

$$
\begin{aligned}
\mathbf{E}\left(\left(D_{1}^{+}\right)^{2}\right)=\frac{\mathbf{E}\left(\left(D_{T}^{+}\right)^{2}\right)}{\mathbf{E}(T)} & =2 \lambda \int_{0}^{\infty} \mathbf{P}\left(D_{T}^{+}>\alpha\right) \alpha d \alpha \\
& =\int_{0}^{\infty} \frac{u}{\operatorname{ch} u} d u=2 \beta(2)
\end{aligned}
$$

Next we determine $\mathbf{E}\left(D_{t}^{+} D_{t}^{-}\right)$. Firstly,

$$
\mathbf{E}\left(D_{1}^{+} D_{1}^{-}\right)=\frac{\mathbf{E}\left(D_{T}^{+} D_{T}^{-}\right)}{\mathbf{E}(T)}=\lambda \int_{0}^{\infty} \int_{0}^{\infty} \mathbf{P}\left(D_{T}^{+}>\alpha, D_{T}^{-}>\beta\right) d \alpha d \beta .
$$


Supposing $\alpha<\beta$ we have from (4.14)

$$
\begin{aligned}
\mathbf{P}\left(D_{T}^{+}<\alpha, D_{T}^{-}<\beta\right) & =1-\frac{1}{\operatorname{ch}(\alpha \sqrt{2 \lambda})}-v(\alpha, \beta) \\
& =\mathbf{P}\left(D_{T}^{+}<\alpha\right)-v(\alpha, \beta)
\end{aligned}
$$

with $v$ as given in (4.15). Consequently,

$$
\mathbf{P}\left(D_{T}^{+}<\alpha, D_{T}^{-}>\beta\right)=v(\alpha, \beta)
$$

and

$$
\mathbf{P}\left(D_{T}^{-}>\beta\right)-\mathbf{P}\left(D_{T}^{+}>\alpha, D_{T}^{-}>\beta\right)=v(\alpha, \beta),
$$

which gives

$$
\mathbf{P}\left(D_{T}^{+}>\alpha, D_{T}^{-}>\beta\right)=\frac{1}{\operatorname{ch}(\beta \sqrt{2 \lambda})}-v(\alpha, \beta), \quad \alpha<\beta .
$$

Hence it holds

$$
\int_{\alpha}^{\infty} \mathbf{P}\left(D_{T}^{+}>\alpha, D_{T}^{-}>\beta\right) d \beta=\int_{\alpha}^{\infty} \frac{1}{\operatorname{ch}(\beta \sqrt{2 \lambda})} d \beta-\int_{\alpha}^{\infty} v(\alpha, \beta) d \beta,
$$

where, by elementary integration,

$$
\int_{\alpha}^{\infty} v(\alpha, \beta) d \beta=\frac{(\operatorname{ch}(\alpha \sqrt{2 \lambda})-1) \operatorname{sh}(\alpha \sqrt{2 \lambda})}{\sqrt{2 \lambda}(\operatorname{ch}(\alpha \sqrt{2 \lambda}))^{2}(\operatorname{ch}(\alpha \sqrt{2 \lambda})+1)}
$$

By symmetry, we have

$$
\begin{aligned}
\mathbf{E}\left(D_{1}^{+} D_{1}^{-}\right) & =2 \lambda\left(\int_{0}^{\infty} d \alpha \int_{\alpha}^{\infty} d \beta \frac{1}{\operatorname{ch}(\beta \sqrt{2 \lambda})}-\int_{0}^{\infty} d \alpha \int_{\alpha}^{\infty} d \beta v(\alpha, \beta)\right) \\
& =\int_{0}^{\infty} \frac{u}{\operatorname{ch} u} d u-\int_{0}^{\infty} \frac{(\operatorname{ch} u-1) \operatorname{sh} u}{\operatorname{ch}^{2} u(\operatorname{ch} u+1)} d u \\
& =2 \sum_{k=0}^{\infty}(-1)^{k}(2 k+1)^{-2}-\int_{1}^{\infty} \frac{x-1}{x^{2}(x+1)} d x \\
& =2 \beta(2)-2 \log 2-1 .
\end{aligned}
$$


Remark 4.10. Recall that

$$
\mathbf{E}\left(T^{p / 2}\right)=\lambda \int_{0}^{\infty} t^{p / 2} \mathrm{e}^{-\lambda t} d t=\Gamma((2+p) / 2) / \lambda^{p / 2} .
$$

With this formula it is now fairly straightforward to connect the pth moment of $D_{1}^{+}$with Dirichlet's $\beta$-function. Indeed, for $p \geq 1$ we have

$$
\begin{aligned}
\mathbf{E}\left(\left(D_{1}^{+}\right)^{p}\right)=\frac{\mathbf{E}\left(\left(D_{T}^{+}\right)^{p}\right)}{\mathbf{E}\left(T^{p / 2}\right)} & =\frac{\lambda^{p / 2} p}{\Gamma\left(\frac{2+p}{2}\right)} \int_{0}^{\infty} \mathbf{P}\left(D_{T}^{+}>\alpha\right) \alpha^{p-1} d \alpha \\
& =\frac{p}{2^{p / 2} \Gamma\left(\frac{2+p}{2}\right)} \int_{0}^{\infty} \frac{t^{p-1} d t}{\operatorname{ch} t} \\
& =\frac{2 p \Gamma(p)}{2^{p / 2} \Gamma\left(\frac{2+p}{2}\right)} \beta(p) .
\end{aligned}
$$

Acknowledgement. We thank Gabor Szekely for posing the problem of finding the covariance of maximum increase and decrease of BM, and Margret Halldorsdottir for observing the connection with Catalan's constant.

\section{References}

[1] M. Abramowitz and I. Stegun. Mathematical functions, 9th printing. Dover publications, Inc., New York, 1970.

[2] J. Bertoin. Lévy Processes. Cambridge University Press, Cambridge, UK, 1996.

[3] Ph. Biane and M. Yor. Valeurs principales associeés aux temps locaux browniens. Bull. Sci. Math., 2e sériè, 111:23-101, 1987.

[4] A.N. Borodin and P. Salminen. Handbook of Brownian Motion - Facts and Formulae, 2nd edition. Birkhäuser, Basel, Boston, Berlin, 2002.

[5] M. Dominé. First passage time distribution of a Wiener process with drift concerning two elastic barriers. J. Appl. Probab., 33:164-175, 1996.

[6] R. Douady, A.N. Shiryayev, and M. Yor. On probability characteristics of "downfalls" in a standard Brownian motion. Theory Prob. Appl., 44:29-38, 1999. 
[7] J.M. Harrison. Brownian Motion and Stochastic Flow Systems. Wiley, New York, 1985.

[8] M. Magdon-Ismail, A.F. Atiya, A. Pratap, and Y.S. Abu-Mostafa. On the maximum drawdown of a Brownian motion. J. Appl. Prob., 41:147161, 2004.

[9] H.P. McKean. Stochastic Integrals. Academic Press, New York, London, 1969.

[10] J. Pitman and M. Yor. Bessel processes and infinitely divisible laws. In D. Williams, editor, Stochastic Integrals, volume 851 of Springer Lecture Notes in Mathematics, pages 285-370, Berlin, Heidelberg, 1981. Springer Verlag.

[11] J. Pitman and M. Yor. A decomposition of Bessel bridges. Z. Wahrscheinlichkeitstheorie verw. Gebiete, 59:425-457, 1982.

[12] D. Revuz and M. Yor. Continuous Martingales and Brownian Motion, 3rd edition. Springer Verlag, Berlin, Heidelberg, 2001.

[13] E. Tanré and P. Vallois. Range of Brownian motion with drift. J. Theor. Probab. (to appear). 